\title{
Federalización e ingresos de investigadores a CONICET en 2019 y 2020: $¿$ Del dicho al hecho hay mucho trecho?
}

Federalization and incorporation of researchers to CONICET in 2019-2020:

Easier said than done?

\section{Andrés Niembro \\ Centro Interdisciplinario de Estudios sobre Territorio, Economía y Sociedad (CIETES) \\ aniembro@unrn.edu.ar}

Francisco Aristimuño

Centro de Estudios en Ciencia,

Tecnología, Cultura y Desarrollo

(CITECDE) y Consejo Nacional

de Investigaciones Científicas y Técnicas

(CONICET)

faristimuno@unrn.edu.ar

\section{Juan Carlos Del Bello}

Centro de Estudios en Ciencia,

Tecnología, Cultura y Desarrollo (CITECDE)

jcdelbello@unrn.edu.ar

Fecha de recepción:

17.9 .20

Fecha de aceptación:

23.12.20

\section{Resumen}

El artículo analiza los ingresos a la Carrera de Investigador Científico y Tecnológico (CIC) del Consejo Nacional de Investigaciones Científicas y Técnicas (CONICET) en 2019 y 2020, en relación con el objetivo de federalización (desconcentración territorial) y a la luz de dos estrategias divergentes que han atravesado históricamente al organismo. El análisis cuantitativo muestra que la federalización de la CIC en las últimas convocatorias ha pasado principalmente por el programa de fortalecimiento, mientras que los resultados en las otras ventanillas sugieren el abandono de los criterios geográficos de años atrás. Esto indica un retorno hacia el enfoque academicista característico de la convocatoria libre con el equilibrio entre las distintas áreas del conocimiento como único criterio adicional.

Palabras clave: políticas de ciencia y tecnología - desconcentración territorial - CONICET - universidades públicas - programa de fortalecimiento a la $\mathrm{I}+\mathrm{D}+\mathrm{i}$. 


\begin{abstract}
This article analyzes the incorporation of new members to the Career of the Scientific and Technological Researcher (CIC) of the CONICET in 2019 and 2020, concerning the objective of federalization (territorial redistribution) and in light of two divergent strategies that have been historically at the core of CONICET. The quantitative analysis shows that the federalization of the CIC in the last two calls has mainly gone through the program for strengthening of $R E D$, while the results in the other modalities suggest the abandonment of the geographical criteria of years ago. This seems to indicate a return to an academic approach, which is characteristic of the general or free call with the balance between the different areas of knowledge as the only additional criterion.
\end{abstract}

Keywords: science and technology policies - territorial redistribution - CONICET - public universities - program for the strengthening of $R E D$.

\title{
1. Introducción
}

Argentina es un país con profundas desigualdades regionales (Cao y Vaca 2006; Niembro 2015) y estas inequidades se ven reflejadas además en la distribución territorial del personal y las inversiones en ciencia y tecnología (CyT). Sin ir más lejos, los últimos planes nacionales de ciencia, tecnología e innovación (CTI) destacan los fuertes desbalances territoriales a raíz de la concentración de recursos en la ciudad y provincia de Buenos Aires, Córdoba y Santa Fe (MINCYT 2011; 2013; SECYT 2006). En el sistema universitario argentino también se observa una elevada aglomeración de alumnos, docentes e investigadores en las principales universidades del centro del país (Jeppesen et al. 2016; Lugones et al.2010) y un cuadro similar puede encontrarse al evaluar el destino geográfico de los fondos administrados por la Agencia Nacional de Promoción Científica y Tecnológica (Lastra 2017; Peirano 2011; Suárez y Fiorentin 2018), o bien la localización de la infraestructura científico-tecnológica (Gutti et al. 2019; MINCYT 2020). Asimismo, la desigual distribución entre provincias de los recursos humanos del Consejo Nacional de Investigaciones Científicas y Técnicas (CONICET) ha sido ampliamente reconocida (CONICET 2006; Gallardo 2015; Jeppesen et al. 2015; Szpeiner y Jeppesen 2013). 
El caso del CONICET es clave pues es el principal organismo del sistema argentino de CyT en términos presupuestarios y tiene por misión "el fomento y ejecución de actividades científicas y tecnológicas en todo el territorio nacional y en las distintas áreas del conocimiento" (Artículo 1 del Decreto 1661/96). En el período reciente, el CONICET ha cobrado aún mayor relevancia, ya que operó como la punta de lanza de la expansión del sistema a partir de 2003 (Albornoz y Gordon 2011; Aliaga 2019; Botto y Bentancor 2018; Cruz Castro et al. 2016). Por ejemplo, la cantidad de investigadores del CONICET creció un 54\% entre 2010 y 2015 , frente a un incremento de apenas 6\% en el número de docentes-investigadores con dedicación exclusiva en el sistema universitario (según datos de los anuarios estadísticos de la Secretaría de Políticas Universitarias). Por otra parte, aunque en las últimas décadas se crearon nuevas universidades públicas en diversas provincias, paralelamente ha crecido la cantidad de instituciones en el conurbano bonaerense (González y Claverie 2017; Marquina y Chiroleu 2015). Como señalan Beigel et al. (2018), muchas de estas universidades mantienen estrechos vínculos con la Ciudad Autónoma de Buenos Aires (CABA), tanto por la migración de docentes e investigadores formados en la Universidad de Buenos Aires (UBA) como por contar con instalaciones de posgrado y/o investigación en la CABA.

La preocupación por la federalización de la CyT en Argentina ha sido histórica y no ha distinguido signo político, desde el primer plan de ciencia y tecnología de 1972 hasta la actualidad. Particularmente en las últimas décadas, la federalización se ha establecido con fuerza en el discurso de políticas del área, ya sea al interior del naciente Ministerio de Ciencia, Tecnología e Innovación (MINCYT) como del CONICET (Emiliozzi y Unzué 2015; Szpeiner y Jeppesen 2013; Unzué 2015). En la actualidad, pareciera existir además cierto consenso entre las principales fuerzas políticas del país sobre la necesidad de profundizar la federalización de la CyT en Argentina (Agencia TSS, 19-09-2019). A pesar de todo esto, los estudios recientes sobre el tema muestran que los progresos han sido modestos, incipientes y dispares entre regiones o provincias (Gallardo 2015; Jeppesen et al. 2015; Niembro 2020a; Sarthou 2019; Unzué y Emiliozzi 2017).

Vale la pena aclarar que la mirada más tradicional en Argentina respecto a la federalización responde a la desconcentración territorial de la inversión y el personal en CyT (Niembro 2020b), y es en este punto donde principalmente se enmarca el artículo. No obstante, también existe una concepción de tipo cualitativa o institucional, vinculada a la descentralización de algunas decisiones y/o funciones en torno a las políticas de CTI. Asimismo, la descentralización puede contener un componente de desconcentración territorial en términos organizacionales. Un ejemplo ha sido la descentralización de algunas funciones administrativas y de gestión del CONICET a partir de la creación de Centros Científicos Tecnológicos (CCT) en distintas regiones (González 2017). De cualquier forma, la autora destaca que los CCT regionales, o la creación de nuevas unidades ejecutoras de investigación y transferencia por parte del CONICET, no han logrado romper con la circularidad y concentración territorial de la estructura de CyT. 
La centralización y concentración del diseño, el financiamiento y las capacidades científico-tecnológicas no es sólo una característica histórica de Argentina, sino que representa un rasgo compartido por buena parte de América Latina. No obstante, en algunos países de la región pueden rastrearse esfuerzos por descentralizar las políticas de CTI y desconcentrar los recursos, aunque con intensidades diferentes, marchas y contramarchas (Casas et al. 2013; Llisteri y Pietrobelli 2011; Rivas et al. 2014). Por ejemplo, el caso de Brasil se ha tomado como una experiencia interesante para extraer lecciones (Albornoz et al. 2015; CEPAL 2017; dos Santos 2017) y también existen algunos antecedentes en México (Casas y Dettmer 2007; Chávez 2008). La implementación de políticas regionales de CTI ha venido creciendo sostenidamente en Europa (GonzálezLópez et al. 2019; Isaksen et al. 2018) e incluso se ha intentado trasladar parte de esta experiencia a Latinoamérica (Barceló et al. 2015).

En este marco, el objetivo del presente artículo es contrastar dichos y hechos recientes en torno a la federalización del CONICET, desde la óptica de la desconcentración territorial, analizando y aportando algunas reflexiones sobre la distribución provincial de los ingresos de investigadores en 2019 y 2020 -es decir, los postulantes seleccionados de las convocatorias abiertas en 2018 y 2019-. Estas dos últimas convocatorias a la carrera del investigador científico y tecnológico (en adelante, CIC) tuvieron la particularidad de ser las primeras en presentar tres ventanillas, al incorporarse el fortalecimiento a la $I+D+i$ a las ventanillas general y de temas estratégicos, que ya venían conviviendo desde 2013. Por otro lado, si bien ambas convocatorias se abrieron bajo el (anterior) gobierno de Mauricio Macri, los resultados de los ingresantes en 2020 se dan tras el cambio de mandato, concomitante con una ampliación en el número de ingresos (de 450 estipulados a 760). Estas particularidades de las últimas convocatorias a la CIC permiten aportar nuevas reflexiones sobre la federalización del CONICET, comparando tanto entre ventanillas como entre diferentes contextos políticos.

Luego de esta introducción, el trabajo se desarrolla en cinco secciones. La sección 2 ofrece un breve marco teórico y metodológico. En la sección 3 se repasa la importancia del CONICET dentro del sistema de CyT, algunas características históricas de la CIC y sus vínculos con el tema de la federalización. Sobre esto último, en la sección 4 se pone de manifiesto el consenso que pareciera existir entre diferentes actores claves del CONICET en torno a la necesidad de una mayor federalización del Consejo. En la sección 5 se presenta un análisis sobre los dos últimos ingresos a la CIC (2019 y 2020), tanto a nivel general como por provincias, lo cual permite examinar el impacto de este instrumento a los fines de la federalización del CONICET. Por último, la sección 6 concluye con una serie de reflexiones finales y discusiones de política. 


\section{Breve marco teórico-metodológico}

Puesto que este artículo busca analizar el diseño e implementación del ingreso a la CIC del CONICET en los años 2019 y 2020, vale la pena aclarar el marco conceptual desde el que se aborda esta política particular. En primer lugar, hay que reconocer que el término política en la lengua española admite múltiples significados (AguilarVillanueva 1992). Esta multiplicidad de significados se ve sustancialmente reducida en la lengua inglesa, que posee tres términos distintos: policy, politics y polity. Sin intención de adentrarnos en el extenso debate alrededor de estos términos, varios autores coinciden en que: politics refiere a la dinámica del juego político; polity al orden del sistema político, a su estructura institucional con sus distintos niveles jurídicos, políticos y administrativos; y finalmente, policy a una línea de conducta asumida por un agente particular, en general, el gobierno (Lahera 2006; Losada Trabada 2003; Vargas Velásquez 1999, entre otros).

En este artículo, el foco estará puesto en el análisis de una política (policy) específica (el ingreso a la CIC del CONICET) en un período determinado (2019-2020). Lo que buscamos con este estudio va en línea con la definición de Dye (2017) del campo de análisis de las políticas, a saber, una investigación sistemática sobre lo que el gobierno hace, por qué lo hace y qué consecuencias se derivan de ello. Por su parte, Aguilar Villanueva (1992) diferencia entre el elemento técnico y el político de toda buena política pública (policy). Esto es, toda política puede ser juzgada desde el punto de vista de su eficiencia instrumental (dimensión técnica) o desde la deseabilidad de su objetivo (dimensión política). En este artículo no se discute la deseabilidad del objetivo de la federalización de la CyT, del CONICET y de los ingresos a CIC en especial, sino que se lo toma como dado en función a las múltiples expresiones públicas de funcionarios en este sentido (los dichos). En términos de Oszlak y O’Donell (1995), esto implica asumir a la federalización como una cuestión, que tiene una historia y que se ha objetivado como un nudo social alrededor del cual gira la hechura de las políticas en el sector.

En este sentido, el artículo pretende estudiar el diseño e implementación de esta política (el ingreso a la CIC del CONICET) en relación al objetivo explícito de la federalización (en tanto desconcentración territorial), para así reflexionar sobre su racionalidad técnica y los resultados efectivamente alcanzados (Britto et al. 2014). El foco no estará puesto en el proceso de construcción de estas políticas, en cómo se definieron los cupos de ingreso a la CIC en cada una de las convocatorias, o en qué actores e intereses influyeron en la conformación de las distintas ventanillas. Si bien son preguntas que quedan por fuera de los alcances de este artículo, a la luz de los resultados que se presentan, sería interesante abordarlas en futuras investigaciones.

La metodología de trabajo se concentra en la recopilación, procesamiento y análisis de datos (a nivel provincial), y su interpretación a la luz de dos estrategias divergentes que 
han atravesado históricamente al CONICET. Dada la escasez de series temporales de indicadores territoriales en materia de CyT, o bien la habitual demora con la que se publica la información estadística, no son tan frecuentes los estudios de esta naturaleza. No obstante, los datos que el CONICET publica en sus informes y actas de gestión o en el portal CONICET en cifras, junto con los que pueden re-construirse a partir de los resultados de las convocatorias, aportan una aproximación a la distribución territorial del sistema argentino de CyT (en línea con Emiliozzi y Unzué 2015).

En este sentido, los listados que publicó el CONICET en 2019 y 2020 al informar los resultados de las últimas convocatorias de ingreso a la CIC en sus diferentes ventanillas son insumos claves para este artículo. En el caso de 2019, se trata del listado de ingresantes a la CIC, y en 2020, tanto de los ingresantes como de los postulantes que quedaron en el orden mérito pero no fueron seleccionados. Un primer punto de análisis general surge del contraste entre el número (agregado) de ingresantes por ventanilla respecto a lo estipulado previamente al momento del anuncio de cada convocatoria. A su vez, estos valores se comparan con los cupos anunciados en la convocatoria 2020 (cuyos ingresantes se conocerán en 2021).

Por otra parte, una particularidad de los resultados de las dos últimas convocatorias a la CIC, a diferencia de años anteriores, es que se publicaron los lugares de trabajo de los postulantes. El procesamiento de estos listados nos permitirá entonces desarrollar otras comparaciones. Una de ellas es el análisis según el tipo de institución que representa el lugar de trabajo propuesto -universidad pública, instituto CONICET, organismo de CyT, entre otros-. Para ello, clasificamos a los ingresantes en 2019 y 2020 siguiendo las categorías que se utilizan en el portal CONICET en cifras y comparamos con algunos valores de años anteriores que provienen de este mismo portal (donde se reflejan datos de todo el universo de la CIC). Pero el punto central de este trabajo, dado el foco puesto en la federalización, es el procesamiento y clasificación de los resultados de los dos últimos ingresos a la CIC en función de la provincia donde se encuentra el respectivo lugar de trabajo. Esta clasificación provincial coincide con la escala geográfica adoptada por varias estadísticas oficiales publicadas en los últimos informes de gestión del Consejo (2018 y 2019) y, sobre todo, con la información disponible en CONICET en cifras, a la que también recurrimos para algunos contrastes.

\section{CONICET, CIC y federalización}

Desde su creación en 1958 y con el pasar de los años, el CONICET se ha establecido como el principal organismo de CyT de Argentina (Albornoz y Gordon 2011; Bekerman 2016; Cruz Castro et al. 2016; Unzué y Emiliozzi 2017), explicando en las últimas décadas alrededor de un tercio del presupuesto público total del área.A su vez, tras las reformas de mediados de los años noventa (Del Bello 2014), la mayoría del presupuesto del CONICET se ha concentrado 
en salarios y estipendios, teniendo como ejes principales a la CIC (10.923 personas en 2019), las becas de doctorado y postdoctorado (10.295 en 2019) y la carrera del personal de apoyo (CPA, con 2.792 en 2019). Según los cálculos de Stefani (2018), el porcentaje del presupuesto del CONICET para cubrir todos los salarios y becas ha rondado entre el $87 \%$ y el $94 \%$ en el periodo 2009-2018, quedando un escaso margen para los gastos operativos o la inversión en equipamiento e infraestructura. Por ello, como resaltan Szpeiner y Jeppesen (2013:8), "su asignación anual de becas e ingresos marca en gran medida el rumbo y las perspectivas de desarrollo científico y tecnológico argentino ya sean en lo temático como en lo geográfico".

No obstante, las diferencias en las remuneraciones entre becarios e investigadores suelen ser significativas (Barrera 2019). Según Arleo et al. (2011), en 2010 el CONICET destinó el $57 \%$ de su presupuesto a la CIC y $26 \%$ a becas (en ese año se registraban 6.350 investigadores y 8.122 becarios). Cálculos propios en base a información presupuestaria del CONICET en 2018, indican que las CIC y CPA (juntas) significaron el 64\% del presupuesto y las becas, otro $24 \%$. Otra diferencia importante entre la carrera del investigador y las becas es la estabilidad laboral, ya que estas últimas duran un periodo de años determinado y no hay garantía de continuidad a través del ingreso a la CIC cuando finalizan. En este sentido, la carrera del investigador no sólo representa la mayor inversión que ejecuta el principal organismo de CyT del país, sino también la que determina de un modo más duradero el perfil temático y regional del Consejo.

Vale la pena recordar que la CIC no siempre fue lo que hoy conocemos. Bernardo Houssay, principal impulsor del Decreto-Ley 1.291/58 que dio creación al CONICET, era partidario de un tipo de CIC de soporte y apoyo a la investigación universitaria, similar a la National Science Foundation de Estados Unidos. En su esquema original, la CIC era un sistema de categorización donde los investigadores recibían estipendios o subsidios por parte del CONICET, mientras mantenían su relación laboral y salarial con el organismo de pertenencia, ya fueran las universidades $\mathrm{u}$ otros organismos nacionales de CyT.Así funcionó la CIC hasta el Decreto-Ley 20.464 del 23 de mayo de 1973 -sólo dos días antes del retorno de la democracia-, el cual aprobó el estatuto de la CIC como estructura laboral y salarial del CONICET. Este no había sido el enfoque original de Bernardo Houssay y Rolando García, Presidente y Vicepresidente del CONICET en su fundación (Caldelari et al. 1992; Hurtado 2010).

Si bien Houssay y García compartían la visión de una CIC que apoyara e incentivara a los recursos humanos mientras mantenían su relación laboral con otras instituciones del sistema de CyT (principalmente, las universidades), al poco tiempo de andar se evidenciaron diferencias entre estas dos personalidades respecto a la estrategia de promoción que debía adoptar el CONICET (Hurtado 2010). Estas diferencias cobran un renovado interés a la luz de este artículo, siendo que muestran la convivencia dentro del organismo de dos estrategias divergentes que, lejos de estar saldadas, continúan hasta la actualidad. 
La mayoría del Directorio original, encabezado por Houssay, pretendía un esquema de promoción donde la excelencia académica fuera el único criterio de otorgamiento, dejando de lado todo intento por priorizar el desarrollo de alguna temática o región específica. Houssay creía que "la mejor manera de tener ciencia aplicada es intensificar la investigación científica fundamental, pues de ella derivarán abundantes aplicaciones" (Houssay 1960:11). En otros términos, sostenía una visión lineal ofertista del cambio tecnológico y la innovación. El segundo grupo, representado en el Directorio del CONICET por García, buscaba fomentar líneas de investigación poco consolidadas en Argentina (como las ciencias sociales) y lograr un esquema más federal, al mismo tiempo que más conectado con el contexto social y económico del país (Caldelari et al. 1992; Hurtado 2010). Desde esta perspectiva, se desconfiaba del impacto del modelo lineal ofertista y se creía que, para alcanzar el desarrollo, era necesario un mayor grado de planificación en el área de CyT. Si bien esta vertiente aún no lograba uniformidad conceptual en los tiempos del primer Directorio del CONICET, apuntaba a lo que luego fue conocido como el Pensamiento Latinoamericano en Ciencia, Tecnología y Sociedad (PLACTS).

En los hechos, el enfoque de Houssay logró imponerse y continuó tras la creación de la CIC con relación laboral y salarial en 1973, por lo que la política del Consejo para el ingreso a la CIC se basó en la evaluación de postulaciones (libre demanda) con base en el mérito académico como criterio principal. Dicho mérito se establece mediante puntajes a cargo de Comisiones Asesoras por áreas y sub-áreas de conocimiento que convergen en la Junta de Calificación. Así, la evaluación de las postulaciones comprende los antecedentes científicos (y tecnológicos) del postulante, la línea de investigación a desarrollar, la idoneidad y trayectoria del Director (y eventualmente Co-director) y las capacidades científico-tecnológicas (en equipamiento y recursos humanos) de la institución donde va a desarrollar sus actividades.

La convocatoria general o libre fue la única modalidad de ingreso a la CIC hasta el Plan Nacional Plurianual de CyT 1999-2001, que incorporó áreas prioritarias o de vacancia de interés nacional o regional y estableció (sin precisar un porcentaje o cantidad) que una parte de los ingresos a CIC (alrededor de 150 por año en total) fueran reservados para esas áreas (GACTEC 1999). De algún modo, esta política buscó recuperar la línea del grupo de García, que consideraba que el CONICET debía promocionar líneas de investigación vacantes y con un sentido federal. No obstante, en general siempre fueron dominantes los ingresos por la convocatoria libre.

En el periodo de recuperación y crecimiento del financiamiento a la CyT que se abrió a partir de 2003-2004, el CONICET se estableció como un organismo clave (Albornoz y Gordon 2011; Aliaga 2019; Bekerman 2016; Botto y Bentancor 2018; Cruz Castro et al. 2016; Emiliozzi y Unzué 2015), puesto que, en un proceso de consolidación institucional, incrementó ampliamente la cantidad de becas e ingresos a carrera. Uno de 
los ejes principales de la política de CyT por ese entonces consistió, precisamente, en el aumento y rejuvenecimiento del personal, ya que luego de varios años de crisis y ajuste se apreciaba un envejecimiento de la base de científicos e investigadores (CONICET 2006; Unzué y Emiliozzi 2017). Entre 2003 y 2010, la CIC pasó de 3.800 investigadores a más de 6.300, un incremento del 66\%. Sin embargo, durante estos años el criterio de áreas prioritarias o de vacancia se restringió al otorgamiento de becas, mientras que el ingreso a la CIC se rigió únicamente por la calidad académica, por disciplina y gran área del conocimiento, y sin importar el destino geográfico (Sarthou 2019). Por ello, varios autores han resaltado que este proceso de recuperación del personal en CyT vino acompañado por un agravamiento de las desigualdades territoriales ya existentes (Jeppesen et al. 2015; Szpeiner y Jeppesen 2013; Unzué 2015; Unzué y Emiliozzi 2017).

A partir de 2010-2011 se produjo un punto de inflexión hacia una nueva etapa (Emiliozzi y Unzué 2015; Unzué y Emiliozzi 2017) donde, en el marco de las políticas de federalización que venía trazando el MINCYT, se tomaron algunas decisiones y se adoptaron criterios geográficos en el CONICET para intentar promover una distribución más federal de las becas y los ingresos a la CIC. Sobre este tema, Unzué (2015:16) detalla que:

el CONICET produce una serie de estudios que servirán de insumos para definir los criterios que se deben adoptar, entre ellos mapeos de recursos humanos para ver su distribución geográfica [...]. En este sentido, se senalaron regiones en las que se detectaba un déficit de investigadores, sea en relación a los becarios o por falta de masa crítica. Esta información llevó a priorizar el ingreso a la carrera de investigadores provenientes de ciertas áreas geográficas en detrimento de las centrales.

Según Szpeiner y Jeppesen (2013), quienes se desempeñaban durante esos años en las áreas de evaluación y planificación del CONICET, a partir de 2010 se desarrolló un conjunto de estudios cuantitativos "que sirvieron como antecedentes para la implementación de nuevas políticas de federalización en las convocatorias anuales a becas e ingresos a la carrera del investigador" (Szpeiner y Jeppesen 2013:6). La propuesta llevó a incorporar "un porcentaje a priori de vacantes para cubrir provincias/localidades prioritarias por disciplinas” (Szpeiner y Jeppesen 2013:10). Las provincias más rezagadas eran consideradas "prioridades de primer orden indistintamente de cuestiones disciplinares", o puesto en otros términos, "en provincias como Chaco o Formosa se consideró deseable el asentamiento de investigadores sin importar su disciplina ya que representaba para estas provincias un valor agregado por sí mismo” (Szpeiner y Jeppesen 2013:14). 
Además de estas incorporaciones, que buscaban tomar en cuenta la cuestión federal, a partir de 2013 los ingresos a la CIC incorporaron una ventanilla adicional por temas estratégicos. Los temas de dicha ventanilla fueron definidos de acuerdo con el Plan Argentina Innovadora 2020 y siguen vigentes hasta la actualidad. Sarthou (2019) muestra que en el período 2013-2016 -en el cual se alcanzó un pico de 914 ingresos en 2015- sólo entre el 9\% y 15\% de los ingresantes, según el año, entraron por temas estratégicos. La nueva ventanilla para temas estratégicos y los criterios geográficos en los ingresos significaron una recuperación, aunque bastante incipiente, de la visión de García para el CONICET como un organismo encargado de promocionar recursos con vistas al desarrollo federal de las capacidades científicas y direccionadas por las áreas prioritarias que se identificaran a nivel nacional y regional.

A partir de 2016, y tras la asunción del gobierno de Mauricio Macri, se dieron nuevas modificaciones en el ingreso a la CIC del CONICET. El primer cambio, ocurrido en 2016, fue la brusca rebaja en el número de ingresos, que se redujo a menos de 400. En dicha convocatoria se respetó la distribución aproximada de $90 \%$ para la ventanilla general y $10 \%$ para temas estratégicos que se venía sosteniendo en años anteriores. Sin embargo, a partir de ese año, se estableció que los ingresos de 2017 serían repartidos de forma equitativa entre ambas ventanillas, fijando un cupo de 300 para cada una (600 en total).

$\mathrm{Al}$ año siguiente se incorporaron nuevos cambios. En primer lugar, a petición del Consejo Interuniversitario Nacional (CIN) se introdujo una modalidad inédita denominada fortalecimiento a la $\mathrm{I}+\mathrm{D}+i$, con el objetivo de favorecer el ingreso de investigadores en universidades públicas (mayoritariamente nacionales) y organismos nacionales de CyT de menor desarrollo científico-tecnológico relativo (excluidos, por ejemplo, el INTA y la CNEA). Como se verá más adelante, las universidades públicas incluidas entre las instituciones a fortalecer representaron el $90 \%$ de estos ingresos. El programa de fortalecimiento abarcó a las universidades que tenían menos de 150 miembros en la CIC, un listado encabezado por la Universidad Nacional del Nordeste (UNNE) con 117 investigadores, y cerrado por universidades que no contaban con ningún investigador del CONICET. A cada institución se le asignaba un cupo de 5 cargos, cuya cobertura resultaba de postulaciones a un número equivalente de líneas prioritarias bajo la denominación de perfiles, que eran propuestos por las instituciones y aprobados por el CONICET previo a la apertura de la convocatoria. En segundo lugar, la convocatoria 2018 (y también la 2019) programó distribuir un total de 450 cargos por partes iguales (tercios) entre la convocatoria general, la orientada a temas estratégicos y el programa de fortalecimiento a la $I+D+I$.

\section{Del dicho: la federalización del CONICET como objetivo de política}

Lograr una distribución más equilibrada de las capacidades de CyT a lo largo y ancho del país ha sido un objetivo de la política del sector desde hace cinco décadas por lo menos. 
El primer plan de CyT, elaborado en 1972, ya lo distinguía como uno de sus objetivos y, desde el retorno de la democracia en 1983, ha sido un factor común a todos los responsables de la cartera de CyT del país. Menciones en este sentido pueden encontrarse en los "Lineamientos para una política científica y Tecnológico" de Sadosky (1984), como así también en los discursos de Matera mientras era Secretario de CyT e interventor del CONICET en los primeros años del gobierno de Menem (Aristimuño y Aguiar 2015; Matera 1992). Asimismo, se pueden encontrar objetivos y políticas concretas en el período de reforma del sistema de CyT encarado por Del Bello entre 1996 y 1999 (Del Bello 2007). El Plan Estratégico de CyT del 2006 y el aún hoy vigente Plan Argentina Innovadora 2020 también consideran a la federalización como un objetivo clave.

En una entrevista realizada en el año 2017, el actual Ministro de CTI, Roberto Salvarezza, señalaba que "federalizar la ciencia fue prioritario durante mi gestión [en el CONICET entre 2012 y 2015], por la concentración que teníamos de recursos humanos en determinadas zonas del país" (Diario Contexto, 03-07-2017). En ese mismo año, el por entonces Presidente del CONICET, Alejandro Cecatto, manifestaba que:

Federalizar el sistema científico argentino es una gran necesidad, por una cuestión de equidad. El 75-80\% de los recursos humanos del CONICET están concentrados en la zona central del país y hay grandes esfuerzos que hacen grupos incipientes -algunos más o menos consolidados pero en general sin toda la masa crítica necesaria- en distintas regiones más alejadas, desde la Patagonia, Noroeste o Noreste del país. Entonces, hemos tomado como decisión del Directorio del CONICET, una decisión importante, muy proactiva en cuanto a la federalización, que ha sido llevar a un 30\% el ingreso a carrera del investigador con posiciones asignadas a estas zonas de vacancia geográfica. Es el porcentaje más alto que la institución ha establecido $y$ pretendemos seguir avanzando en la medida que esos cupos vayan generando una distribución más equitativa (Diario La Gaceta, 09-07-2017).

En efecto, el porcentaje mencionado es un poco superior al que originalmente se planteaba en el llamado "Plan Barañao 2013", el cual establecía que "se apuntará a la federalización de los recursos humanos priorizando el 25\% de las vacantes del CONICET en aquellas regiones del país con escaso desarrollo científico" (Hurtado 2016:s/p). No obstante, la experiencia suele mostrar que la política de cupos por sí sola ha sido relativamente incapaz para revertir las demás tendencias del sistema a la concentración territorial. Al respecto, en el año 2013, Santiago Sacerdote -por entonces Director y 
Vicepresidente de Asuntos Tecnológicos del CONICET- señalaba que:

En los últimos diez años, el CONICET está creciendo a una tasa muy importante, del 10\%, lo que implica que ingresen en 2013 a la carrera 720 investigadores (...). Hay un cupo por estas restricciones, y la demanda es mayor, por lo que van a entrar por orden de mérito $y$, a partir de determinado lugar, ingresarán quienes vengan de zonas no centrales (...), pero lo cierto es que de lugares no centrales no se presenta la suficiente cantidad de gente, así que, aunque uno haga una reserva del 25\% para zonas no centrales, los que se presentan no llegan al 10\% (...). Queremos llevar la matriz de distribución geográfica hacia algo distinto y con esto sólo no alcanza

(Agencia Télam, 03-08-2013).

Otra experiencia con algunas similitudes puede rastrearse en 1997, previo a la normalización del CONICET -elección de los miembros en representación de la comunidad científica-. Durante la intervención de Del Bello en el Consejo, la reapertura de la carrera del investigador estableció un cupo de vacantes para siete regiones del país. Los postulantes podían presentar dos opciones de lugares de trabajo, por lo que la competencia por méritos estaba restringida a cada región. Esto significaba que un postulante con méritos medios, que no hubiera alcanzado a ingresar entre los cupos de alguna de las regiones que históricamente concentraron el mayor número de investigadores de CONICET, sí podía hacerlo en una región de bajo desarrollo relativo. Cuando el cupo no se cubría en una región (como fue el caso del NEA, por ejemplo), las vacantes no cubiertas se distribuían proporcionalmente en el resto de las regiones, hasta agotar los ingresos para un máximo total de cargos. A este método se lo conoció como la calesita, ya que el procedimiento tenía varias vueltas hasta la cobertura total de las vacantes. No obstante, a las regiones metropolitana y centro del país ingresaron más investigadores que el cupo predeterminado, lo cual indica que no necesariamente una política focalizada logra los resultados esperados en materia científico-tecnológica, ya que el desarrollo de capacidades en CyT es un proceso evolutivo y muchas veces desigual.

En parte por las limitaciones antes señaladas, Dora Barrancos -Directora del CONICET entre 2010 y 2019- reconocía en una entrevista que "una cuenta pendiente es profundizar la federalización del sistema; no logramos acertar políticas ni promover mejores acuerdos con las instituciones provinciales para ubicar investigadores en las zonas geográficas en las que hay vacancia” (Diario Página12, 12-05-2019). De igual forma, la actual Presidenta del CONICET, Ana Franchi, ha destacado a la federalización como una de las deudas al interior del Consejo (Agencia Télam, 18-01-2020), además de resaltar entre las prioridades del 
área "federalizar, pero federalizar en serio" (Agencia TSS, 19-09-2019). Salvarezza señalaba recientemente que "las asimetrías estructurales que existen entre las regiones de nuestro país precisan un desarrollo científico y tecnológico realmente federal" (Portal AgendAR, 10-10-2019), mientras que Mario Pecheny -actual Director y Vicepresidente de Asuntos Científicos del CONICET- destacaba la necesidad de "plantear en serio un crecimiento equilibrado del CONICET en todo el territorio” (Portal UNERnoticias, 07-05-2019).

El informe público de gestión del Consejo al 31-12-2018 destaca que para fortalecer las capacidades en CyT "se pone el énfasis en la promoción de la federalización" (CONICET 2018:11). Entre las prioridades que fija el informe, se encuentra "orientar las convocatorias (carrera y becas)", contemplando "la distribución entre investigación fundamental, temas estratégicos y tecnología así como también la distribución más equitativa entre todas las provincias del país" (CONICET 2018:14). Argumentos similares pueden encontrarse entre las líneas de acción propuestas por Miguel Laborde y Tulio Del Bono -Directores por el área de ciencias agrarias e ingenierías y por los organismos provinciales de CyT, respectivamente-:"revisar los criterios de ingreso a la CIC y al sistema de becas en función de prioridades disciplinares y geográficas"; “ "federalizar la ciencia y la tecnología, dándole mayor equidad a la distribución regional de recursos” (Laborde y Del Bono 2019:10).

Por último, Ana Franchi ha señalado recientemente que "hay provincias ganadoras y perdedoras, y eso hay que solucionarlo" (Portal Política Argentina, 02-10-2019). Entre sus prioridades al frente del CONICET figuran "federalizar ese organismo para que no exista concentración solo en las grandes ciudades y bajar la ciencia al territorio, una acción que permite resolver los problemas del país” (Agencia Télam, 18-01-2020).

\section{A los hechos: ingresos a la CIC de CONICET en 2019 y 2020}

\subsection{Análisis general de los resultados}

Un primer contrapunto general respecto a las últimas convocatorias a la CIC puede trazarse entre lo proyectado al momento de su apertura (2018 y 2019) y lo consumado al anunciarse los resultados en 2019 y 2020, respectivamente. Como puede apreciarse en el Cuadro 1, en 2019 ingresó en total a la CIC prácticamente la misma cantidad de investigadores que estaba pautada. Sin embargo, en los hechos no se concretó el anunciado reparto por tercios, sino que se traspasó el 40\% de las vacantes estipuladas para fortalecimiento (sólo 88 ingresos) a la ventanilla general (208 ingresantes), totalizando así un 46\% de los cupos para temas libres, un $34 \%$ para temas estratégicos y sólo un 20\% para la nueva ventanilla de fortalecimiento. En 2020, tras el cambio de gobierno, se definió un importante incremento en la cantidad de ingresos a la CIC (de 450 a 760), pero esta ampliación tampoco se distribuyó por tercios. En cambio, se optó por consolidar a la ventanilla general, que representó así el 56\% de los ingresantes (10 p.p. más que en 2019), en detrimento de las ventanillas de temas estratégicos y fortalecimiento, que terminaron teniendo el $23 \%$ y $21 \%$ de los ingresos, respectivamente. 
Cuadro 1. Ingresos en 2019 y 2020 por ventanilla: proyectado vs. ejecutado

\begin{tabular}{|c|c|c|c|c|c|c|c|c|c|c|c|c|}
\hline & \multicolumn{3}{|c|}{$\begin{array}{c}\text { Proyectado en Convocatorias } \\
\text { (2018 y 2019) }\end{array}$} & \multicolumn{4}{c|}{ Ejecutado en Ingreso 2019 } & \multicolumn{3}{c|}{ Ejecutado en Ingreso 2020 } \\
\cline { 2 - 13 } & General & $\begin{array}{c}\text { Temas } \\
\text { Estrat. }\end{array}$ & $\begin{array}{c}\text { Fortal. } \\
\text { I+D+i }\end{array}$ & Total & General & $\begin{array}{c}\text { Temas } \\
\text { Estrat. }\end{array}$ & $\begin{array}{c}\text { Fortal. } \\
\text { I+D+i }\end{array}$ & Total & General & $\begin{array}{c}\text { Temas } \\
\text { Estrat. }\end{array}$ & $\begin{array}{c}\text { Fortal. } \\
\text { I+D+i }\end{array}$ & Total \\
\hline No.1 & 150 & 150 & 150 & 450 & 208 & 153 & 88 & 449 & 429 & 172 & 159 & 760 \\
\hline$\%$ del total & 33,3 & 33,3 & 33,3 & 100 & 46,3 & 34,1 & 19,6 & 100 & 56,4 & 22,6 & 20,9 & 100 \\
\hline $\begin{array}{l}\% \text { cambio } \\
\text { ingreso- } \\
\text { convoc. }\end{array}$ & & & & & 38,7 & 2 & $-41,3$ & 0 & 186 & 14,7 & 6 & 68,9 \\
\hline
\end{tabular}

Fuente: Elaboración propia.

Los números anunciados respecto a la convocatoria 2020 (cuyos resultados estarán en 2021) tienden a consolidar los porcentajes consumados en los últimos ingresos a la CIC. La mitad de los 800 cargos anunciados se destinará a la convocatoria general, de temática libre, y la mitad restante se distribuirá entre diferentes ventanillas orientadas, abandonándose la idea de repartos equitativos (los tercios).

El programa de fortalecimiento tendrá 160 vacantes para universidades públicas (exactamente, el 20\%) y otros 20 cupos (2,5\%) serán para organismos de CyT. A su vez, se anunció que se modifica el criterio para la selección de las universidades participantes del programa y de los perfiles solicitados, con el objetivo adicional, a partir de esta convocatoria, de procurar una distribución armónica entre las cuatro grandes áreas del conocimiento. Es decir, se abandona el criterio previo de contar con menos de 150 investigadores en la CIC (en total y en cualquier área) para reemplazarlo por un criterio que contempla la situación de cada área del conocimiento en cada institución. Así, serán elegibles universidades que cuenten con menos de 40 miembros de la CIC en ciencias agrarias e ingenierías, o en ciencias sociales y humanidades; y 50 investigadores en ciencias biológicas y de la salud, o en ciencias exactas y naturales. Por ello, en el listado de universidades aparecen ahora otras 8 instituciones por encima de la UNNE, entre las cuales tres corresponden a la provincia de Buenos Aires, una a Córdoba y otra a Santa Fe.

Entre las otras modalidades orientadas, la ventanilla de temas estratégicos se reducirá a apenas 100 cargos, lo cual implicaría una caída sostenida en su peso relativo en los últimos años (34\% de los ingresos de 2019, 23\% en 2020, y 12,5\% proyectado para 2021), retornando así a la participación que tuvo en el período 2013-2016. Por último, se establece un número nada despreciable de 120 vacantes (15\%) para Proyectos Especiales y Convocatoria Permanente desde el Exterior, una modalidad descripta, por ahora, de forma bastante laxa: "ingresos especiales a determinar durante el año, incluyendo tanto aquellos ingresos en convocatoria permanente desde el exterior como convocatorias 
específicas que tengan por objeto promover y/o consolidar el desarrollo científico y tecnológico en determinadas disciplinas, temáticas, localizaciones, instituciones, áreas geográficas de vacancia y/o prioritarias, proyectos especiales que se consideren estratégicos o necesidades de determinadas Unidades, etc.".

Llegados a este punto, cabe destacar que las últimas decisiones tomadas en el CONICET no necesariamente contaron con el aval de todos los miembros del Directorio. Entre las voces más críticas, que se opusieron a los últimos cambios y propusieron otra distribución alternativa para la convocatoria 2020, se encuentran Del Bono y Laborde, quienes en una nota de marzo de 2020 expresan que

el incremento en el número de ingresos, no es precisamente para favorecer el federalismo sino para favorecer a los grupos concentrados en las principales ciudades y universidades del país. (...) Aumentar los ingresos a Temas Libres supone aceptar la plena vigencia del Modelo Lineal, cuestión que ya no se acepta en ninguna parte del mundo. Supone también aceptar que si dejamos todo librado a la demanda de los ingresantes (...), una "mano invisible" dispondrá que esos ingresos vayan, necesariamente, a atender las necesidades más apremiantes de la sociedad (Del Bono y Laborde 2020:4)

Otro aspecto general de la CIC que resulta interesante analizar es la distribución de los investigadores y de los últimos ingresantes según el tipo de institución que establecen como su lugar de trabajo. Como puede observarse en el Cuadro 2, entre 2007 y 2018 ha aumentado considerablemente el peso de los institutos de CONICET, en gran medida por la expansión y el crecimiento de los institutos con contraparte de universidades. La contracara de esta tendencia de la última década ha sido la caída en la participación de investigadores de la CIC en centros exclusivamente de universidades públicas. Mientras que en 2007 los pesos de estas dos categorías eran similares, en 2018 los investigadores en institutos CONICET prácticamente triplicaban a los de (solamente) universidades públicas. Puede apreciarse además que los últimos ingresos por la ventanilla general y por temas estratégicos tienden a replicar las mismas proporciones. Lo contrario ocurre, previsiblemente, con el programa de fortalecimiento, donde aumenta considerablemente el peso de los centros universitarios y de los organismos de CyT como lugares de trabajo, a pesar de que en algunas universidades los institutos fortalecidos son de dependencia compartida con el CONICET. 
Cuadro 2. Investigadores de la CIC e ingresos por tipo de institución (en porcentaje)

\begin{tabular}{|l|c|c|c|c|c|c|c|c|c|c|c|c|}
\hline \multirow{2}{*}{ Tipo de lugar de trabajo } & \multicolumn{3}{|c|}{ Total de la CIC } & \multicolumn{3}{|c|}{ Ingresos 2019 } & \multicolumn{3}{c|}{ Ingresos 2020 } \\
\cline { 2 - 13 } & $\mathbf{2 0 0 7}$ & $\mathbf{2 0 1 7}$ & $\mathbf{2 0 1 8}$ & General & $\begin{array}{c}\text { Temas } \\
\text { Estrat. }\end{array}$ & $\begin{array}{c}\text { Fortal. } \\
\text { I+D+i }\end{array}$ & Total & General & $\begin{array}{c}\text { Temas } \\
\text { Estrat. }\end{array}$ & $\begin{array}{c}\text { Fortal. } \\
\text { I+D+i }\end{array}$ & Total \\
\hline $\begin{array}{l}\text { Institutos CONICET con contraparte en } \\
\text { Universidades y OCyT o Exclusivos CONICET }\end{array}$ & 45,2 & 66,3 & 68,9 & 68,3 & 60,8 & 30,7 & 58,4 & 66,9 & 66,3 & 23,3 & 57,6 \\
\hline Universidades de Gestión Pública & 42,5 & 24,5 & 23,9 & 23,6 & 25,5 & 54,5 & 30,3 & 26,1 & 26,2 & 67,3 & 34,7 \\
\hline Organismos de Ciencia y Técnica & 6,0 & 5,0 & 3,3 & 5,8 & 7,8 & 14,8 & 8,2 & 3,3 & 5,2 & 9,4 & 5,0 \\
\hline Otros (empresa, fundación, asociación civil) & 3,8 & 2,4 & 2,2 & 1,4 & 4,6 & 0 & 2,2 & 1,4 & 1,2 & 0 & 1,1 \\
\hline Universidades de Gestión Privada & 2,5 & 1,8 & 1,7 & 1,0 & 1,3 & 0 & 0,9 & 2,3 & 1,2 & 0 & 1,6 \\
\hline
\end{tabular}

Fuente: Elaboración propia.

Hay al menos dos cuestiones que se desprenden del análisis anterior y que no podemos dejar de observar. En primer lugar, la resistencia al cambio en la cultura institucional del CONICET, que tras repetidos intentos en su historia por integrar criterios que complementen al mérito académico, siempre retorna a priorizar la ventanilla libre o general con el equilibrio entre las distintas áreas del conocimiento como único criterio adicional. El último de estos intentos fue el de repartir equitativamente los ingresos entre las distintas ventanillas y sumar un programa específico de fortalecimiento a organismos con bajo nivel de desarrollo relativo. Las resistencias del CONICET a estos cambios se pueden ver manifestadas en las diferencias entre los ingresos estipulados (en 2018 y 2019) y los efectivos en 2019 y 2020. Finalmente, el anuncio de la convocatoria 2020 termina por consolidar, una vez más, a la ventanilla general como la principal vía de acceso a la CIC del CONICET.

En segundo lugar, un aspecto que resulta de suma relevancia para el desarrollo futuro del complejo nacional de CyT es la creciente participación de la CIC en la nómina de investigadores de otras instituciones, en especial, de las universidades nacionales. La CIC del CONICET se ha convertido en el medio por el cual muchas universidades logran aumentar el número de investigadores en su institución (Aliaga 2019; MINCYT 2018). Recordemos que, por ejemplo, la cantidad de investigadores del CONICET creció un 54\% entre 2010 y 2015 y la de docentes investigadores con dedicación exclusiva en el sistema universitario sólo un 6\%. Como se ha señalado además, la gran mayoría de los nuevos investigadores de la CIC suele asentarse en institutos de doble pertenencia entre el CONICET y alguna universidad, o en menor medida, en algún centro universitario -y este segundo tipo de lugar de trabajo es naturalmente más fuerte en la ventanilla de fortalecimiento-.

\subsection{Un análisis provincial de los últimos ingresos a la CIC}

Pasemos ahora a analizar el impacto territorial de los ingresos a la CIC en 2019 y 2020 a través de las diferentes ventanillas. Como puede apreciarse en el Cuadro 3, los primeros 
cuatro lugares son los esperados: ciudad y provincia de Buenos Aires, Córdoba y Santa Fe. Luego aparecen Río Negro (explicado básicamente por la ciudad de Bariloche), Mendoza y Tucumán, aunque en 2019 estas dos últimas provincias fueron superados por Entre Ríos. En general, las restantes provincias logran representar menos del 2\% o incluso del 1\% de los ingresos año a año. En la parte media e inferior de la tabla puede observarse además que en varias provincias es común que no haya ingresantes en alguna/s de las ventanillas, lo que en el extremo llevó a la ausencia de ingresos en Corrientes y Formosa en 2019. La ventanilla en la que más se redujeron estos ceros fue la de fortalecimiento, que pasó de tener 7 provincias sin ingresantes en 2019 a sólo 2 en 2020. Asimismo, la federalización de la CIC en estos años, en tanto ingreso de nuevos investigadores en provincias de menor desarrollo relativo, parece haber pasado principalmente por el canal de fortalecimiento. Incluso, este fenómeno se ha acentuado de una convocatoria a otra, ya que en 2020 esta ventanilla da cuenta de la totalidad de los ingresantes en 7 provincias y del $50 \%$ o más en otras 6 (última columna).

Aunque se trata de un razonamiento contrafáctico, podemos suponer que, de no haberse abierto esta ventanilla específica, varias de las provincias más rezagadas del país hubieran incorporado una menor cantidad de investigadores o tal vez no hubieran tenido ningún ingresante a la CIC. No obstante, también hay que reconocer que el número de ingresos por fortalecimiento en dichas provincias parece ser relativamente acotado como para, por sí solo, reducir o acortar las brechas existentes (como se verá luego). 
Cuadro 3. Ingresos a la CIC-CONICET en 2019 y 2020 por provincia y ventanilla (ordenado por total de ingresantes en 2020)

\begin{tabular}{|c|c|c|c|c|c|c|c|c|c|c|c|c|c|c|c|c|c|c|}
\hline & \multicolumn{9}{|c|}{ Ingresos 2019} & \multicolumn{9}{|c|}{ Ingresos 2020} \\
\hline & \multicolumn{2}{|c|}{ General } & \multicolumn{2}{|c|}{\begin{tabular}{|c|} 
Temas \\
Estratégicos
\end{tabular}} & \multicolumn{2}{|c|}{$\underset{I+D+i}{\text { Fortalecim. }}$} & \multicolumn{2}{|c|}{ Total } & \multirow{2}{*}{\begin{tabular}{|c|}
$\begin{array}{c}\text { Fortal } \\
\text { / Total }\end{array}$ \\
$\%$ \\
\end{tabular}} & \multicolumn{2}{|c|}{ General } & \multicolumn{2}{|c|}{\begin{tabular}{|c|} 
Temas \\
Estratégicos
\end{tabular}} & \multicolumn{2}{|c|}{$\underset{I+D+i}{\text { Fortalecim. }}$} & \multicolumn{2}{|c|}{ Total } & \multirow{2}{*}{\begin{tabular}{|c|c|}
$\begin{array}{c}\text { Fortal } \\
\text { / Total }\end{array}$ \\
$\%$ \\
\end{tabular}} \\
\hline & No. & $\%$ & No. & $\%$ & No. & $\%$ & No. & $\%$ & & No. & $\%$ & No. & $\%$ & No. & $\%$ & No. & $\%$ & \\
\hline Buenos Aires & 56 & 26,92 & 52 & 33,99 & 33 & 37,50 & 141 & 31,40 & 23,40 & 120 & 27,97 & 61 & 35,47 & 50 & 31,45 & 231 & 30,39 & 21,65 \\
\hline CABA & 67 & 32,21 & 39 & 25,49 & 8 & 9,09 & 114 & 25,39 & 7,02 & 142 & 33,10 & 26 & 15,12 & 14 & 8,81 & 182 & 23,95 & 7,69 \\
\hline Córdoba & 32 & 15,38 & 15 & 9,80 & 5 & 5,68 & 52 & 11,58 & 9,62 & 49 & 11,42 & 19 & 11,05 & 8 & 5,03 & 76 & 10,00 & 10,53 \\
\hline Santa Fe & 23 & 11,06 & 19 & 12,42 & 1 & 1,14 & 43 & 9,58 & 2,33 & 37 & 8,62 & 23 & 13,37 & 4 & 2,52 & 64 & 8,42 & 6,25 \\
\hline \begin{tabular}{|l} 
Río Negro \\
\end{tabular} & 9 & 4,33 & 5 & 3,27 & 3 & 3,41 & 17 & 3,79 & 17,65 & 19 & 4,43 & 7 & 4,07 & 6 & 3,77 & 32 & 4,21 & 18,75 \\
\hline Mendoza & 5 & 2,40 & 5 & 3,27 & 0 & 0 & 10 & 2,23 & 0 & 21 & 4,90 & 7 & 4,07 & 0 & 0 & 28 & 3,68 & 0 \\
\hline Tucumán & 4 & 1,92 & 3 & 1,96 & 1 & 1,14 & 8 & 1,78 & 12,50 & 10 & 2,33 & 6 & 3,49 & 0 & 0 & 16 & 2,11 & 0 \\
\hline \begin{tabular}{|l|} 
San Juan \\
\end{tabular} & 1 & 0,48 & 1 & 0,65 & 0 & 0 & 2 & 0,45 & 0 & 4 & 0,93 & 5 & 2,91 & 6 & 3,77 & 15 & 1,97 & 40,00 \\
\hline Misiones & 0 & 0 & 1 & 0,65 & 3 & 3,41 & 4 & 0,89 & 75,00 & 1 & 0,23 & 5 & 2,91 & 8 & 5,03 & 14 & 1,84 & 57,14 \\
\hline \begin{tabular}{|l|} 
Jujuy \\
\end{tabular} & 1 & 0,48 & 2 & 1,31 & 4 & 4,55 & 7 & 1,56 & 57,14 & 1 & 0,23 & 3 & 1,74 & 9 & 5,66 & 13 & 1,71 & 69,23 \\
\hline Chubut & 1 & 0,48 & 1 & 0,65 & 3 & 3,41 & 5 & 1,11 & 60,00 & 8 & 1,86 & 2 & 1,16 & 3 & 1,89 & 13 & 1,71 & 23,08 \\
\hline \begin{tabular}{|l|} 
Salta \\
\end{tabular} & 2 & 0,96 & 2 & 1,31 & 5 & 5,68 & 9 & 2,00 & \begin{tabular}{|l|}
55,56 \\
\end{tabular} & 2 & 0,47 & 3 & 1,74 & 7 & 4,40 & 12 & 1,58 & 58,33 \\
\hline Corrientes & 0 & 0 & 0 & 0 & 0 & 0 & 0 & 0 & 0 & 6 & 1,40 & 0 & 0 & 6 & 3,77 & 12 & 1,58 & 50,00 \\
\hline Entre Ríos & 0 & 0 & 5 & 3,27 & 8 & 9,09 & 13 & 2,90 & 61,54 & 0 & 0 & 0 & 0 & 8 & 5,03 & 8 & 1,05 & 100 \\
\hline \begin{tabular}{|l|} 
San Luis \\
\end{tabular} & 2 & 0,96 & 0 & 0 & 0 & 0 & 2 & 0,45 & 0 & 4 & 0,93 & 2 & 1,16 & 1 & 0,63 & 7 & 0,92 & 14,29 \\
\hline T del Fuego & 0 & 0 & 1 & 0,65 & 0 & 0 & 1 & 0,22 & 0 & 3 & 0,70 & 0 & 0 & 3 & 1,89 & 6 & 0,79 & 50,00 \\
\hline La Pampa & 1 & 0,48 & 0 & 0 & 2 & 2,27 & 3 & 0,67 & 66,67 & 2 & 0,47 & 0 & 0 & 3 & 1,89 & 5 & 0,66 & 60,00 \\
\hline La Rioja & 1 & 0,48 & 0 & 0 & 2 & 2,27 & 3 & 0,67 & 66,67 & 0 & 0 & 0 & 0 & 5 & 3,14 & 5 & 0,66 & 100 \\
\hline Neuquén & 1 & 0,48 & 1 & 0,65 & 0 & 0 & 2 & 0,45 & 0 & 0 & 0 & 3 & 1,74 & 2 & 1,26 & 5 & 0,66 & 40,00 \\
\hline Chaco & 1 & 0,48 & 0 & 0 & 5 & 5,68 & 6 & 1,34 & 83,33 & 0 & 0 & 0 & 0 & 4 & 2,52 & 4 & 0,53 & 100 \\
\hline Catamarca & 1 & 0,48 & 1 & 0,65 & 1 & 1,14 & 3 & 0,67 & 33,33 & 0 & 0 & 0 & 0 & 4 & 2,52 & 4 & 0,53 & 100 \\
\hline Sgo. del Estero & 0 & 0 & 0 & 0 & 3 & 3,41 & 3 & 0,67 & 100 & 0 & 0 & 0 & 0 & 4 & 2,52 & 4 & 0,53 & 100 \\
\hline Santa Cruz & 0 & 0 & 0 & 0 & 1 & 1,14 & 1 & 0,22 & 100 & 0 & 0 & 0 & 0 & 2 & 1,26 & 2 & 0,26 & 100 \\
\hline Formosa & 0 & 0 & 0 & 0 & 0 & 0 & 0 & 0 & 0 & 0 & 0 & 0 & 0 & 2 & 1,26 & 2 & 0,26 & 100 \\
\hline Total País & 208 & 100 & 153 & 100 & 88 & 100 & 449 & 100 & 19,60 & 429 & 100 & 172 & 100 & 159 & 100 & 760 & 100 & 20,92 \\
\hline
\end{tabular}

Fuente: Elaboración propia.

Si bien la federalización no ha sido en principio un objetivo explícito de la ventanilla de fortalecimiento, tal y como fue concebida, claramente una parte de los ingresos a la CIC por fortalecimiento ha contribuido a este objetivo en los últimos años. Decimos una parte pues no hay que perder de vista que Buenos Aires, CABA y Córdoba representaron en 2019 el 52\% y en 2020 el 45\% de los ingresos por fortalecimiento, ayudando a fortalecer algunos organismos de CyT o universidades de escaso -y por ende necesario- desarrollo científico en estas provincias centrales. Por otro lado, dado que muchas de las universidades comprendidas en el programa de fortalecimiento se encuentran igualmente en provincias de menor desarrollo científico-tecnológico relativo, es que una porción de los ingresantes por dicha ventanilla ha aportado de forma implícita a la federalización de la CIC. En este sentido, el Consejo Interuniversitario Nacional (CIN) ha celebrado esta iniciativa "en virtud de que tiende a morigerar desequilibrios y contribuye al desarrollo de la ciencia con un sentido federal y cooperativo", según consta en la Nota 80024 del 5 de abril de 2019, dirigida al Presidente del CONICET -donde se reclama por el mencionado recorte del $40 \%$ en los ingresos efectivos por fortalecimiento-.

El rol que parece haber tenido la ventanilla de fortalecimiento de cara a la federalización en las últimas convocatorias a la CIC se acrecienta si miramos el subtotal de ingresantes 
por las otras dos ventanillas, es decir, la general más temas estratégicos (Cuadro 4). Un primer aspecto llamativo es que, en 2020, a pesar del incremento en el número de vacantes, 7 provincias no obtienen ningún ingreso en general o temas estratégicos, pero 4 de ellas sí habían incorporado investigadores en 2019 por alguna de estas ventanillas -en Corrientes pasa lo contrario-. En la comparación entre años, cae un poco el peso relativo de las primeras cuatro jurisdicciones (de 84\% en 2019 a 79\% en 2020), especialmente por una menor participación de Córdoba y Santa Fe en los ingresos. Esta menor concentración se compensa en gran medida con el crecimiento de Mendoza, Río Negro y Tucumán, mientras que el peso de las últimas 17 provincias se mantiene muy bajo (sube de 7,5\% a 9\%). No obstante, hacia el interior de este grupo hay ganadores ( valor de la última columna mayor a 1) y perdedores (menor a 1 ) en el paso de una convocatoria a otra.

Cuadro 4. Ingresos por las ventanillas general y temas estratégicos (sin fortalecimiento) en 2019 y 2020 , por provincia

\begin{tabular}{|c|c|c|c|c|c|}
\hline & \multicolumn{2}{|c|}{2019} & \multicolumn{2}{|c|}{2020} & \multirow{2}{*}{\begin{tabular}{|l} 
Ratio \\
$\% 2020$ / \\
$\% 2019$
\end{tabular}} \\
\hline & No. & $\%$ & No. & $\%$ & \\
\hline Primer grupo (4) & 303 & 83,93 & 477 & 79,37 & 0,95 \\
\hline Buenos Aires & 108 & 29,92 & 181 & 30,12 & 1,01 \\
\hline CABA & 106 & 29,36 & 168 & 27,95 & 0,95 \\
\hline Córdoba & 47 & 13,02 & 68 & 11,31 & 0,87 \\
\hline Santa Fe & 42 & 11,63 & 60 & 9,98 & 0,86 \\
\hline Segundo grupo (3) & 31 & 8,59 & 70 & 11,65 & 1,36 \\
\hline Mendoza & 10 & 2,77 & 28 & 4,66 & 1,68 \\
\hline Río Negro & 14 & 3,88 & 26 & 4,33 & 1,12 \\
\hline Tucumán & 7 & 1,94 & 16 & 2,66 & 1,37 \\
\hline Restantes 17 & 27 & 7,48 & 54 & 8,99 & 1,20 \\
\hline Chubut & 2 & 0,55 & 10 & 1,66 & 3,00 \\
\hline San Juan & 2 & 0,55 & 9 & 1,50 & 2,70 \\
\hline San Luis & 2 & 0,55 & 6 & 1,00 & 1,80 \\
\hline Misiones & 1 & 0,28 & 6 & 1,00 & 3,60 \\
\hline Corrientes & 0 & 0 & 6 & 1,00 & - \\
\hline Salta & 4 & 1,11 & 5 & 0,83 & 0,75 \\
\hline Jujuy & 3 & 0,83 & 4 & 0,67 & 0,80 \\
\hline Neuquén & 2 & 0,55 & 3 & 0,50 & 0,90 \\
\hline Tierra del Fuego & 1 & 0,28 & 3 & 0,50 & 1,80 \\
\hline La Pampa & 1 & 0,28 & 2 & 0,33 & 1,20 \\
\hline Entre Ríos & 5 & 1,39 & 0 & 0 & 0 \\
\hline Catamarca & 2 & 0,55 & 0 & 0 & 0 \\
\hline Chaco & 1 & 0,28 & 0 & 0 & 0 \\
\hline La Rioja & 1 & 0,28 & 0 & 0 & 0 \\
\hline Formosa & 0 & 0 & 0 & 0 & - \\
\hline Santa Cruz & 0 & 0 & 0 & 0 & - \\
\hline Santiago del Estero & 0 & 0 & 0 & 0 & - \\
\hline Total País & 361 & 100 & 601 & 100 & \\
\hline
\end{tabular}

Fuente: Elaboración propia.

¿La ausencia o el bajo número de ingresantes en varias de las provincias relativamente rezagadas se debe a que no se presentaron postulantes (con méritos) en estas provincias? En función de los datos que nos aportan los resultados de la última convocatoria (Cuadro 5), la respuesta es: no necesariamente. Si bien en algunas provincias no hay 
candidatos con mérito suficiente y, por ende, tampoco ingresantes, son varias las provincias rezagadas que no registran ingresos a pesar de tener postulantes en el orden de mérito (gris oscuro), más aún en la ventanilla de temas estratégicos. Algo similar ocurre en otras provincias rezagadas que logran algunos ingresos, pero a su vez tienen el mismo número o más de postulantes con mérito que quedan afuera de los seleccionados (gris intermedio). Cabe destacar la cantidad de casos como estos entre los temas estratégicos, ya que de haber ingresado estos investigadores se hubieran matado dos pájaros de un tiro desde la óptica de Rolando García: se contribuiría a la resolución de problemas prioritarios, fomentando además la radicación en provincias de menor desarrollo científicotecnológico. En este sentido, los resultados parecen indicar que no se estarían siguiendo actualmente algunos de los criterios mencionados en años anteriores, como el de definir a priori un porcentaje para las zonas de vacancia geográfica (Szpeiner y Jeppesen 2013) o de "entrar por orden de mérito y, a partir de determinado lugar, (...) quienes vengan de zonas no centrales" (Agencia Télam, 03-08-2013). En cambio, los resultados publicados de la última convocatoria estarían reflejando principalmente la aplicación del criterio academicista, sin importar tanto el lugar de trabajo de los candidatos. En este contexto, como señalaba el propio Salvarezza en la entrevista de 2017:

la mayoría de los cargos va a quedar en manos de aquellos lugares que tengan más desarrollo $y$, por ende, los más competitivos. En un sistema donde prima la meritocracia, va a ganar el más fuerte. Esta competencia darwiniana va a acrecentar la centralización

(Diario Contexto, 03-07-2017).

Esto último se vislumbra claramente en la convocatoria general o libre, donde las primeras cuatro provincias explican el 81\% de los ingresos y, al agregar las tres siguientes, se alcanza el 93\%. En una entrevista de este año, Diego Hurtado -actual Secretario de Planeamiento y Políticas de CTI- resaltaba

El desequilibrio geográfico del sector de ciencia y tecnología es apabullante (...). Alguien que dirige un instituto importante en la región metropolitana me decía: Hay más investigadores en este laboratorio que en todo el noreste argentino

(Agencia TSS, 24-01-2020).

Entonces, ¿de dónde se espera que salgan las propuestas más potentes y con mayores posibilidades de éxito en el proceso competitivo?, ¿de una gran universidad o instituto del área central o de algún pequeño y posiblemente naciente centro de investigaciones de una región periférica? Esto último nos permite revalorizar lo que significa que un postulante de una provincia rezagada haya logrado entrar en el orden de mérito, aunque 
no así alcanzar el puntaje del grupo de elite que quedó en los primeros puestos seleccionados. Las dos últimas columnas del Cuadro 5 ofrecen medidas alternativas que podrían tomarse para analizar el grado de productividad de postulantes con mérito, en función del número de investigadores o becarios postdoctorales en la provincia. Si bien en muchas de las provincias rezagadas estos indicadores son bajos, en algunas otras superan a la norma nacional o incluso a los valores de provincias centrales. Formosa es muy especial, por su bajísimo número de investigadores y becarios, pero pueden destacarse los casos de Misiones, San Juan, Corrientes, Neuquén o Chaco, según el indicador que se mire.

Cuadro 5. Ingresantes y postulantes con mérito por las ventanillas general y temas estratégicos en 2020, por provincia (ordenado por total)

\begin{tabular}{|c|c|c|c|c|c|c|c|c|c|c|c|c|}
\hline & \multicolumn{4}{|c|}{ General } & \multicolumn{4}{|c|}{ Temas Estratégicos } & \multirow{2}{*}{\multicolumn{4}{|c|}{$\begin{array}{l}\text { Total de postulantes } \\
\text { con Mérito }\end{array}$}} \\
\hline & \multicolumn{2}{|c|}{$\begin{array}{c}\text { Ingresantes } \\
\text { (seleccionados) }\end{array}$} & \multicolumn{2}{|c|}{$\begin{array}{c}\text { Otros con Mérito } \\
\text { (no seleccionados) }\end{array}$} & \multicolumn{2}{|c|}{$\begin{array}{l}\text { Ingresantes } \\
\text { (seleccionados) }\end{array}$} & \multicolumn{2}{|c|}{$\begin{array}{c}\text { Otros con Mérito } \\
\text { (no seleccionados) }\end{array}$} & & & & \\
\hline & No. & $\%$ & No. & $\%$ & No. & $\%$ & No. & $\%$ & No. & $\%$ & \begin{tabular}{|c|} 
c/100 \\
CIC 2019 \\
\end{tabular} & \begin{tabular}{|l} 
c/10 Post- \\
Doc 2019 \\
\end{tabular} \\
\hline Buenos Aires & 120 & 27,97 & 66 & 26,72 & 61 & 35,47 & 68 & 31,05 & 315 & 29,52 & 9,87 & 4,48 \\
\hline CABA & 142 & 33,10 & 85 & 34,41 & 26 & 15,12 & 35 & 15,98 & 288 & 26,99 & 9,46 & 4,99 \\
\hline Córdoba & 49 & 11,42 & 25 & 10,12 & 19 & 11,05 & 25 & 11,42 & 118 & 11,06 & 9,25 & 3,92 \\
\hline Santa $\mathrm{Fe}$ & 37 & 8,62 & 19 & 7,69 & 23 & 13,37 & 22 & 10,05 & 101 & 9,47 & 10,40 & 4,81 \\
\hline \begin{tabular}{|l|} 
Mendoza \\
\end{tabular} & 21 & 4,90 & 11 & 4,45 & 7 & 4,07 & 9 & 4,11 & 48 & 4,50 & 12,50 & 4,85 \\
\hline Tucumán & 10 & 2,33 & 15 & 6,07 & 6 & 3,49 & 10 & 4,57 & 41 & 3,84 & 11,85 & 3,94 \\
\hline Río Negro & 19 & 4,43 & 1 & 0,40 & 7 & 4,07 & 10 & 4,57 & 37 & 3,47 & 7,99 & 4,40 \\
\hline Chubut & 8 & 1,86 & 5 & 2,02 & 2 & 1,16 & 14 & 6,39 & 29 & 2,72 & 13,62 & 6,30 \\
\hline Misiones & 1 & 0,23 & 3 & 1,21 & 5 & 2,91 & 9 & 4,11 & 18 & 1,69 & 22,78 & 3,27 \\
\hline San Juan & 4 & 0,93 & 1 & 0,40 & 5 & 2,91 & 5 & 2,28 & 15 & 1,41 & 12,71 & 2,68 \\
\hline San Luis & 4 & 0,93 & 6 & 2,43 & 2 & 1,16 & 0 & 0 & 12 & 1,12 & 7,50 & 3,64 \\
\hline Salta & 2 & 0,47 & 3 & 1,21 & 3 & 1,74 & 3 & 1,37 & 11 & 1,03 & 8,40 & 2,39 \\
\hline Corrientes & 6 & 1,40 & 1 & 0,40 & 0 & 0 & 2 & 0,91 & 9 & 0,84 & 9,57 & 3,46 \\
\hline Neuquén & 0 & 0 & 1 & 0,40 & 3 & 1,74 & 2 & 0,91 & 6 & 0,56 & 11,54 & 3,33 \\
\hline T del Fuego & 3 & 0,70 & 2 & 0,81 & 0 & 0 & 0 & 0 & 5 & 0,47 & 8,93 & 2,78 \\
\hline Jujuy & 1 & 0,23 & 0 & 0 & 3 & 1,74 & 0 & 0 & 4 & 0,37 & 5,97 & 1,11 \\
\hline La Pampa & 2 & 0,47 & 1 & 0,40 & 0 & 0 & 0 & 0 & 3 & 0,28 & 5,66 & 2,00 \\
\hline Chaco & 0 & 0 & 2 & 0,81 & 0 & 0 & 1 & 0,46 & 3 & 0,28 & 7,89 & 5,00 \\
\hline Catamarca & 0 & 0 & 0 & 0 & 0 & 0 & 1 & 0,46 & 1 & 0,09 & 4,76 & 0,48 \\
\hline Entre Ríos & 0 & 0 & 0 & 0 & 0 & 0 & 1 & 0,46 & 1 & 0,09 & 1,15 & 0,50 \\
\hline Formosa & 0 & 0 & 0 & 0 & 0 & 0 & 1 & 0,46 & 1 & 0,09 & 25,00 & - \\
\hline Sgo. del Estero & 0 & 0 & 0 & 0 & 0 & 0 & 1 & 0,46 & 1 & 0,09 & 2,94 & 0,48 \\
\hline La Rioja & 0 & 0 & 0 & 0 & 0 & 0 & 0 & 0 & 0 & 0 & 0 & 0 \\
\hline Santa Cruz & 0 & 0 & 0 & 0 & 0 & 0 & 0 & 0 & 0 & 0 & 0 & 0 \\
\hline Total País & 429 & 100 & 247 & 100 & 172 & 100 & 219 & 100 & 1067 & 100 & 9,77 & 4,26 \\
\hline
\end{tabular}

Fuente: Elaboración propia.

Finalmente, en el Cuadro 6 se puede apreciar qué efectos tuvieron los dos últimos ingresos (2019 y 2020) en el conjunto de la CIC, tanto en términos absolutos como relativos. En general y como era de esperarse, los cambios han sido marginales y se mantienen enormes brechas entre provincias. Las últimas 17 provincias (de Chubut a Formosa) representaban el 11,3\% del total en 2018 y en 2020 llegarían recién al 11,8\%. Mientras que en la CABA hay un investigador de CONICET cada 1.000 habitantes (o 100 cada 100.000), en Formosa apenas puede encontrarse uno cada 100.000, o 4 en Chaco y Santiago del Estero. Otro aspecto interesante es que, a pesar de la reducción en el peso de la CABA en el total -siendo superada en los últimos 
años por la provincia de Buenos Aires-, de todas formas siguió aumentando el número de investigadores por habitante en la ciudad. Sacando los casos de Tierra del Fuego y Buenos Aires -por el bajo y el muy alto número de habitantes, respectivamente-, las restantes 14 provincias que se ubican al fondo del ranking absoluto son las mismas que en el ranking relativo. Aunque entre estas provincias rezagadas hay ciertos avances relativos año a año -en el orden de más o menos 1 investigador cada 100.000 habitantes-, esto es insuficiente para cerrar las brechas existentes, ya que varias de las primeras provincias han crecido en igual o mayor medida.

Cuadro 6. Investigadores de la CIC por provincia, en \% del total y cada 100.000 habitantes (ordenado por valores 2020)

\begin{tabular}{|l|c|c|c|c|l|c|c|c|c|}
\hline \multicolumn{9}{|c|}{ Investigadores (\%) } & \multicolumn{3}{c|}{ Investigadores cada 100.000 habitantes } \\
\hline Provincia & $\mathbf{2 0 1 0}$ & $\mathbf{2 0 1 8}$ & $\mathbf{2 0 1 9}$ & $\mathbf{2 0 2 0}$ & Provincia & $\mathbf{2 0 1 0}$ & $\mathbf{2 0 1 8}$ & $\mathbf{2 0 1 9}$ & $\mathbf{2 0 2 0}$ \\
\hline Buenos Aires & 28,0 & 29,1 & 29,2 & 29,3 & CABA & 69,7 & 97,3 & 100,9 & 106,7 \\
\hline CABA & 33,4 & 28,1 & 28,0 & 27,7 & Río Negro & 37,8 & 61,1 & 62,6 & 66,1 \\
\hline Córdoba & 11,1 & 11,7 & 11,7 & 11,6 & T del Fuego & 20,5 & 35,8 & 35,5 & 38,1 \\
\hline Santa Fe & 8,5 & 8,8 & 8,8 & 8,8 & Chubut & 17,3 & 34,9 & 35,2 & 36,7 \\
\hline Río Negro & 3,9 & 4,2 & 4,2 & 4,2 & Córdoba & 20,9 & 33,7 & 34,8 & 36,4 \\
\hline Mendoza & 3,3 & 3,6 & 3,5 & 3,5 & San Luis & 18,5 & 31,5 & 31,5 & 32,5 \\
\hline Tucumán & 3,4 & 3,2 & 3,2 & 3,1 & Santa Fe & 16,5 & 26,7 & 27,8 & 29,4 \\
\hline Chubut & 1,4 & 2,0 & 1,9 & 1,9 & Tucumán & 14,6 & 20,7 & 20,9 & 21,6 \\
\hline San Luis & 1,3 & 1,5 & 1,4 & 1,4 & Mendoza & 11,6 & 19,4 & 19,7 & 21,0 \\
\hline Salta & 0,9 & 1,2 & 1,2 & 1,2 & Buenos Aires & 11,3 & 18,0 & 18,6 & 19,7 \\
\hline San Juan & 0,8 & 1,2 & 1,1 & 1,2 & San Juan & 7,5 & 16,4 & 16,4 & 18,2 \\
\hline Corrientes & 0,8 & 0,9 & 0,8 & 0,9 & La Pampa & 6,4 & 13,9 & 14,6 & 15,9 \\
\hline Misiones & 0,3 & 0,7 & 0,7 & 0,8 & Jujuy & 3,5 & 8,1 & 8,9 & 10,5 \\
\hline Entre Ríos & 0,3 & 0,7 & 0,7 & 0,8 & Salta & 4,4 & 8,9 & 9,5 & 10,2 \\
\hline Jujuy & 0,4 & 0,6 & 0,6 & 0,7 & Neuquén & 5,6 & 8,7 & 8,8 & 9,5 \\
\hline T del Fuego & 0,4 & 0,6 & 0,5 & 0,6 & Corrientes & 5,2 & 8,4 & 8,3 & 9,3 \\
\hline Neuquén & 0,5 & 0,5 & 0,5 & 0,5 & La Rioja & 2,6 & 5,7 & 6,4 & 7,6 \\
\hline La Pampa & 0,3 & 0,5 & 0,5 & 0,5 & Misiones & 1,8 & 6,2 & 6,4 & 7,5 \\
\hline Chaco & 0,4 & 0,3 & 0,4 & 0,4 & Entre Ríos & 1,4 & 5,1 & 6,0 & 6,6 \\
\hline Sgo. del Estero & 0,1 & 0,3 & 0,3 & 0,3 & Catamarca & 1,9 & 4,9 & 5,6 & 6,5 \\
\hline La Rioja & 0,1 & 0,2 & 0,2 & 0,3 & Santa Cruz & 3,6 & 4,6 & 4,8 & 5,2 \\
\hline Catamarca & 0,1 & 0,2 & 0,2 & 0,2 & Sgo. del Estero & 0,9 & 3,2 & 3,5 & 3,9 \\
\hline Santa Cruz & 0,2 & 0,2 & 0,2 & 0,2 & Chaco & 2,1 & 2,8 & 3,3 & 3,6 \\
\hline Formosa & 0,06 & 0,04 & 0,04 & 0,05 & Formosa & 0,7 & 0,7 & 0,7 & 1,0 \\
\hline Total País & $\mathbf{1 0 0}$ & $\mathbf{1 0 0}$ & $\mathbf{1 0 0}$ & $\mathbf{1 0 0}$ & Total País & $\mathbf{1 5 , 5}$ & $\mathbf{2 3 , 9}$ & $\mathbf{2 4 , 6}$ & $\mathbf{2 6 , 1}$ \\
\hline
\end{tabular}

Fuente: Elaboración propia. ${ }^{\star}$ Valores 2020 son estimados, adicionando los ingresos al último dato oficial (2019).

\section{Reflexiones finales}

La federalización de la CyT en Argentina ha sido una preocupación histórica y un objetivo de política desde hace varias décadas, especialmente en lo que respecta a la desconcentración territorial de los recursos y capacidades científico-tecnológicas (cuestión a la que pretende contribuir este artículo). Asimismo, en los últimos años la federalización pareciera haber ganado un lugar estratégico en la agenda política del área y, más específicamente, del CONICET -si nos regimos por las manifestaciones de diferentes actores clave y documentos institucionales-. En la última década, el CONICET ha jugado además un papel protagónico en el sistema argentino de CyT, ya que gran parte 
de la expansión del personal científico-tecnológico ha pasado por la política de becas e ingresos de investigadores al Consejo. En este período se habrían adoptado, aparentemente, algunos criterios geográficos para favorecer una distribución más federal y equitativa de las becas y la CIC. En paralelo, una parte (si bien cambiante en el tiempo) del incremento de los recursos humanos en CyT se ha inclinado al tratamiento de temas prioritarios o estratégicos según el último plan de CTI vigente.

Ambos aspectos remiten a una confrontación histórica en el seno del CONICET:la tensión entre una cultura academicista, que impulsa la libre determinación de las temáticas a estudiar -incluso en ausencia de un nexo con la docencia universitaria- y confia en un modelo lineal ofertista del cambio tecnológico y la innovación; y otra que, sin desmerecer la búsqueda de la excelencia, considera estratégico priorizar temas con relevancia nacional o regional, como así también favorecer un desarrollo más federal de la CyT. Históricamente, en el CONICET ha primado el primero de los enfoques. A pesar de ciertos vaivenes temporarios e intentos de incorporar prioridades temáticas y regionales que vayan más allá del criterio general de equilibrio entre las cuatro grandes áreas disciplinares, esta cultura ha mostrado su capacidad de resistencia y siempre ha logrado que el organismo retorne hacia la priorización de la convocatoria libre para la CIC, como ha quedado de manifiesto en los dos últimos ingresos y en los anuncios de la convocatoria 2020.

En este sentido, y tomando en cuenta la magnitud de las brechas territoriales y las diferencias en el grado de desarrollo de distintas áreas temáticas en el sistema argentino de CyT, nos preguntamos: ¿es correcto seguir aplicando reglas homogéneas a contextos heterogéneos?, ¿una universidad o centro de investigaciones periférico y en formación puede regirse por los mismos criterios que una de las grandes universidades o institutos de la zona central?, ¿es posible combinar meritocracia y criterios de excelencia con federalización, comprendiendo las realidades particulares de las zonas rezagadas por su propia condición de tales? O como planteaba Salvarezza -y como han mostrado ampliamente los estudios sobre el tema-, en la competencia "van a ganar los que tengan los grupos más fuertes, porque harán las propuestas más desarrolladas" (Diario Contexto, 03-07-2017). Hasta ahora el CONICET se ha mostrado incapaz de encontrar un equilibrio entre estas cuestiones y algunas de sus últimas decisiones parecen ir en el sentido opuesto. Un ejemplo claro puede encontrarse en la aparente ausencia (o abandono en la aplicación) de los criterios geográficos en los ingresos en 2020 por la ventanilla general y por temas estratégicos, ya que puede apreciarse un número nada despreciable de candidatos de provincias rezagadas en el orden de mérito pero que no llegan a ser seleccionados. Algo parecido sucede con los temas estratégicos, que en 2021 retornarían a niveles similares a los del período 2013-2016.

Respecto a la federalización, el vaso medio lleno, en el contexto antes descripto, es que la ventanilla de fortalecimiento parece consolidarse con al menos un $20 \%$ de las vacantes para la 
CIC. Como se ha visto, la federalización de la CIC en los últimos años ha pasado mayoritariamente por esta ventanilla, ya que una parte de los ingresos (alrededor de la mitad) se da en universidades públicas y organismos nacionales ubicados en provincias relativamente atrasadas o no tradicionales, es decir, por fuera de las cuatro principales jurisdicciones del centro del país.

El vaso medio vacío en cuanto al programa de fortalecimiento es que en las dos últimas convocatorias no se cumplió, en los hechos, con el estipulado reparto equitativo (por tercios) de los ingresos. En este marco, aquella mitad de las vacantes por fortalecimiento -o el equivalente al 10\% del total de ingresos a la CIC- que ha contribuido a la radicación de investigadores en provincias relativamente rezagadas representa un volumen muy limitado e insuficiente para resolver las grandes inequidades territoriales del sistema argentino de CyT, o, en otros términos, para federalizar en serio al CONICET. Asimismo, en la convocatoria 2020 se han cambiado los criterios de selección de las universidades alcanzadas por el programa y de los perfiles solicitados por las mismas, incorporándose por primera vez en esta ventanilla el criterio adicional de la paridad por áreas de conocimiento. Por un lado, esto limita la aplicación de una mirada inter o trans-disciplinar de la CyT en los proyectos institucionales. Por otro, también podría restringir las capacidades de planificación (y progreso) de las universidades que no necesariamente cuentan con todo el abanico de carreras de grado y/o grupos de investigación -como ocurre, por ejemplo, con la escasa cobertura territorial de las carreras ligadas a la salud y las ciencias médicas- y que no podrán seguir expandiendo más allá de cierto límite otras áreas que sí son estratégicas para su proyecto institucional y, posiblemente también, para su contexto territorial. Por último, los nuevos criterios amplían el número de universidades en la competencia por una cantidad similar de cargos, siendo además que varias de las que se suman en la convocatoria 2020 son universidades de la zona central del país, con cierta historia y grado de desarrollo relativo.

Un punto incierto respecto a la federalización en el marco de la convocatoria 2020, y que merecerá ser analizado en el futuro, es el impacto que puedan tener las 120 vacantes (15\% del total) para Proyectos Especiales y Convocatoria Permanente desde el Exterior. En la medida en que al menos una parte de estos cupos (habrá que ver cuánto) se destine a promover la localización de nuevos investigadores en zonas de vacancia geográfica, podría contribuir efectivamente con la federalización de la CIC, más allá de lo que sería esperable que continúe aportando la ventanilla de fortalecimiento.

En suma, vemos problemas tanto en el diseño como en la implementación de las políticas en el sector. Los sesgos a favor de la ventanilla libre observados en las distintas convocatorias, donde parte de los cupos originalmente estipulados para otras ventanillas fueron transferidos a la general, ponen de manifiesto las dificultades para implementar cambios en una estructura con prácticas enraizadas a lo largo de décadas. Los últimos cambios en la convocatoria 2020 muestran, a su vez, problemas en el diseño que 
contradicen los objetivos explícitos de federalización. En este sentido, se evidencian contramarchas en políticas que mostraron ser un canal (aunque incipiente) en pos del objetivo de desconcentración, sin proponer alternativa alguna ni aclarar cómo es que se va a sustanciar la federalización en serio del CONICET.

Llegados a este punto, vale destacar que la federalización de la CyT en Argentina no puede, ni debiera tampoco, restringirse únicamente a los ingresos de nuevos investigadores a la CIC del CONICET, aunque creemos que todavía es mucho lo que se puede corregir y avanzar en este instrumento específico. Un ejemplo de algo que ha quedado por fuera del tratamiento específico de este artículo, pero que resulta un aspecto clave, es la promoción de una mayor movilidad inter-institucional e idealmente también inter-regional, en especial para el grueso de los recursos humanos en CyT que se forman en la zona central del país. Como señala Gallardo (2015:126), en Argentina "la trayectoria de formación e inserción institucional de los investigadores presenta rasgos de escasa movilidad", ya que se verifica "una marcada tendencia a que los investigadores se doctoren en el país y en la misma institución o región donde realizaron previamente sus estudios de grado y en la que se insertarán posteriormente al momento de ingresar al CONICET" (Gallardo 2015:121). ${ }^{1}$

En definitiva, el funcionamiento del propio sistema permite -y en cierto punto favorece o premia- a las trayectorias de grado-doctorado-postdoctorado-investigación en una misma institución, mientras que las reglas del juego en organismos similares de otros países pueden condicionar de antemano una mayor movilidad -por ejemplo, limitando a veces la posibilidad de que los postulantes a investigadores se presenten al mismo lugar donde se formaron a nivel doctoral-. Si se tiene en cuenta que la enorme mayoría de la oferta de posgrado en Argentina se concentra en el área metropolitana de Buenos Aires y en algunas otras universidades del centro del país (Jeppesen et al. 2016), la clave pasaría por generar incentivos que promuevan una mayor desconcentración territorial en las instancias postdoctorales y en el ingreso a la CIC de quienes hicieron su trayectoria previa en la zona central -como se apuntó con los criterios geográficos, que aumentaban las posibilidades de ingresar en zonas de vacancia geográfica-. Asimismo, criterios de este tipo podrían favorecer también el ingreso a la CIC (y la permanencia en el territorio) de aquellos que desarrollaron sus becas de posgrado y/o tuvieron la posibilidad de realizar sus estudios doctorales en provincias relativamente rezagas.

Puesto que en los últimos años el grueso de la federalización en el caso de la CIC se ha circunscripto al programa de fortalecimiento, la relación que se teje entre el CONICET y las universidades es otra cuestión central. Además, en los últimos tiempos las universidades han recalado en la CIC como uno de los medios principales para incrementar su número de investigadores, ante la ausencia de instrumentos de política universitaria que promuevan la incorporación de dedicaciones completas de docencia e investigación. Por otro lado, se observa un marcado crecimiento de la proporción de investigadores de la CIC en institutos 
de doble pertenencia por sobre los centros únicamente universitarios, aunque la ventanilla de fortalecimiento en los dos últimos ingresos a la CIC ayudó a moderar parcialmente esta dinámica. Estas tendencias no serían un problema si las universidades contaran, a su vez, con partidas presupuestarias suficientes para hacerse de los recursos humanos necesarios para cubrir los cargos de docentes-investigadores según su planificación de crecimiento. No obstante, si continúa y se afianza la lógica de que la CIC sea el principal medio para dotarse de investigadores, entonces podría generarse un conflicto entre las prioridades de selección del CONICET y las de cada universidad. En este sentido, ¿¿deben las universidades aceptar el criterio general del CONICET y sumar investigadores en las áreas donde sean más competitivos, sin importar sus requerimientos de recursos para la docencia o su planificación de crecimiento institucional?, o ¿debe el CONICET adaptarse a las necesidades de recursos humanos de las universidades y ayudarlas a conseguir los recursos más capacitados en las áreas prioritarias fijadas por ellas mismas? En el caso de las universidades de menor desarrollo relativo, ¿alcanza con los cinco cupos del programa de fortalecimiento? Está claro que estas cuestiones invitan a una reflexión mucho más amplia que la que aquí se puede ofrecer y dan pie a futuras investigaciones.

Finalmente, otro aspecto clave es la infraestructura necesaria para el despliegue de actividades científicas y tecnológicas a lo largo y ancho del territorio, una dimensión donde también las provincias rezagadas suelen correr por detrás a los grandes institutos y universidades del centro del país (MINCYT 2020). Por ende, el desarrollo de capacidades no puede acotarse a la re-localización de ingresantes a la CIC, sino que entre otras múltiples acciones requiere también del desarrollo de la infraestructura y el equipamiento científico para este personal. La problemática en torno a este punto se agrava si, como se ha señalado, la enorme mayoría del presupuesto del CONICET se destina sólo a salarios y estipendios, dejando un margen muy acotado para, en el mejor de los casos, sostener algunos gastos operativos y ni hablar de nuevas y grandes inversiones. Está claro que sin mayores (y más sostenidos) recursos, la federalización de la CyT se vuelve un objetivo muy dificil de lograr. No obstante, un mayor presupuesto es una condición necesaria pero no suficiente para el logro de objetivos más ambiciosos (Unzué y Emiliozzi 2017). En este sentido, se puede contar con más o menos recursos, pero si los incentivos siguen haciendo que su destino principal sea la zona central del país, poco podrá alcanzarse en cuanto a la federalización de la CyT.

En términos históricos, una de las políticas de federalización más potentes en el caso del CONICET fue la creación y desarrollo de los Centros Regionales durante la dictadura militar iniciada en 1976 -con recursos del primer préstamo BID para el sistema nacional de CyT-: CENPAT en Puerto Madryn, CADIC en Ushuaia, CRIBABB en Bahía Blanca, CRICYT en Mendoza y CERIDE en Santa Fe. Sin embargo, esta política se llevó a cabo en el marco del terrorismo de Estado y tuvo como correlato el divorcio del CONICET de las universidades nacionales, con el consiguiente debilitamiento de las últimas. Más recientemente en el tiempo, una política de desarrollo institucional en esta línea ha sido la creación -de forma conjunta entre el CONICET y distintas universidades- de Centros 
de Investigaciones y Transferencia (CIT) en provincias y ciertas localizaciones en las que el Consejo no contaba con presencia. No obstante, para contribuir más fuertemente con la federalización de la infraestructura de CyT, el programa de los CIT debiera ocupar un lugar mucho mayor en la política (y en los recursos) del CONICET.

Desde el plano de la descentralización institucional (cuestión que no ha sido el foco de este artículo), puede aprovecharse la experiencia de los CCT regionales, en el sentido de construcción de nuevas instituciones de CyT, aunque es necesario todavía remover obstáculos para un funcionamiento sistémico de este tipo de redes. Asimismo, considerando la jerarquización del papel de las universidades públicas a partir de la recuperación de la democracia y la amplia cobertura territorial del sistema universitario argentino, entendemos que toda nueva institucionalidad debiera también apuntar a contemplar a las universidades bajo modalidades innovadoras -como han analizado, por ejemplo, Sanz Menéndez et al. (2011) y Cruz Castro y Sanz Menéndez (2018) en Europa-.

En síntesis, son varios los aspectos que deben abordarse de cara a una política integral de federalización del CONICET. Siguiendo la popular frase de Albert Einstein, "no esperes resultados diferentes, si siempre haces lo mismo". Ojalá este artículo pueda contribuir al debate sobre algunas de las reformas necesarias de la CIC del CONICET, si efectivamente se pretende fomentar la federalización del Consejo y reducir el trecho entre dichos y hechos. 


\begin{abstract}
${ }^{1}$ Los autores agradecen los valiosos comentarios de Gabriela Starobinsky a una versión previa, al igual que las sugerencias de dos evaluadores anónimos. Como es usual, los errores remanentes son de nuestra exclusiva responsabilidad. ${ }^{2}$ Un ejemplo de ello es que, a pesar de los cambios organizacionales ocurridos en los últimos años, con la rebaja a fines de 2018 del rango de Ministerio a Secretaría y la reciente restitución a Ministerio, ha subsistido dentro del organigrama del área la sub/secretaría de federalización de la CyT.
\end{abstract}

${ }^{3} \mathrm{Si}$ bien sería interesante poder analizar hacia el interior de las distintas provincias, esta información no está disponible. Por otro lado, vale destacar que en las provincias de menor desarrollo relativo el CONICET tiende a promover el asentamiento de becarios e investigadores con independencia de la localidad, como señalan Szpeiner y Jeppesen (2013) y como se observa en las matrices geográficas publicadas en las últimas convocatorias a becas.

${ }^{4}$ Rolando García, también Decano de la Facultad de Ciencias Exactas, Físicas y Naturales de la UBA, se exilió en México luego de la intervención de las universidades con el golpe de 1966 y Bernardo Houssay falleció en 1971. ${ }^{5}$ Cabe mencionar que todos los años se registra además un número de vacantes como resultado de jubilaciones, renuncias y fallecimientos. La cobertura de estas vacantes suele seguir los criterios meritocráticos de la convocatoria general, por lo que resulta una ampliación encubierta de los ingresos a la CIC por la convocatoria general, a partir del corrimiento del orden de mérito.

${ }^{6}$ La primera convocatoria (2018) del programa de fortalecimiento adoleció de un grave defecto: sólo ingresaba un investigador por cada perfil, aquél que tuviera más méritos científicos expresado en un mayor puntaje. En el supuesto que para una línea no se postulara nadie o los postulantes no reunieran el puntaje suficiente, la misma quedaba vacante y la institución no cubría el cupo de 5 cargos. Las universidades, claro está, tienen el incentivo de evitar esta situación, por lo que muchas veces presentan perfiles que podrían cubrirse con candidatos potenciales de afuera o de la misma institución. En el último caso, el ingreso a la CIC puede implicar una liberación de presupuesto para la universidad, que puede asignar para atraer a otros docentes-investigadores con criterios diferentes a los de CONICET, o bien a elevar la dedicación de otros docentes de la institución.

${ }^{7}$ Todas estas iniciativas se dieron, a su vez, en el marco de un importante incremento presupuestario para las universidades nacionales, afectado a actividades de CyT. En efecto, el presupuesto para la función de CyT en 2018 (\$650 millones, a valores constantes en términos de 2012) fue 3,5 veces superior al de 2017 (\$150 millones a precios constantes), sin contabilizar los recursos para el Programa de Incentivos a los Docentes Investigadores, que registran un estipendio por investigador en valores nominales igual al establecido cuando se creó el programa en 1994, por lo que su presupuesto sólo crece al compás del aumento del número de docentes investigadores. ${ }^{8}$ Téngase en cuenta que el equilibrio entre áreas, aunque se busca en el agregado de las otras ventanillas, no se impone como condición para la incorporación de nuevos investigadores en cada universidad. Es decir, mientras se establece este nuevo criterio sólo para el programa de fortalecimiento, los ingresos por la ventanilla general pueden seguir permitiendo estructuras desequilibradas. Por ejemplo, la UBA obtuvo 207 ingresantes a la CIC en 2020, pero 88 de estos cargos (el 42,5\%) se concentran en la Facultad de Ciencias Exactas y Naturales, mientras que a Ingeniería sólo se incorporan 4 investigadores, a Veterinaria otros 4, o a Ciencias Económicas 1.

${ }^{9}$ Vale mencionar que entre estas universidades se encuentra también la Universidad Nacional del Comahue, que a pesar de superar los 150 investigadores del CONICET ha sido parte de la convocatoria 2019 y se han seleccionado tres ingresantes en 2020 para fortalecer algunas sedes regionales o grupos menos desarrollados. ${ }^{10} \mathrm{El}$ anuncio puede consultarse en el sitio web de CONICET. Más adelante habrá que ver cuántos de estos cupos terminan efectivamente favoreciendo la localización de nuevos investigadores en zonas de vacancia geográfica.

${ }^{11}$ Esto se encuentra reflejado en las Actas de Directorio 517, 518 y 519, disponibles en el sitio web de CONICET.

${ }^{12}$ Los resultados dados a conocer en 2019 -pero no los de 2020, ni lo publicado en CONICET en cifras- nos permiten desagregar la primera categoría de institutos CONICET e identificar que casi el 90\% de los ingresantes en esta categoría corresponde precisamente a institutos compartidos con universidades.

${ }^{13}$ La relación entre el CONICET y las universidades a través de los institutos de doble dependencia merece un análisis mucho más pormenorizado que el aquí podemos darle -y claramente representa una línea a profundizar-, ya que estos vínculos muchas veces están cargados de diferentes conflictos: a quién responden efectivamente estos institutos, quién evalúa (o no) su desempeño, quién (o en qué proporción) se hace cargo de su funcionamiento, entre otros aspectos (Aliaga 2019; Laborde y Del Bono 2019).

${ }^{14} \mathrm{~A}$ diferencia de los docentes-investigadores con dedicación exclusiva de las universidades, los investigadores de la CIC del CONICET no tienen la responsabilidad, sino la opción, de la docencia. En la convocatoria 2020, pero sólo en el programa de fortalecimiento, las universidades deben indicar, al momento de solicitar un perfil, los eventuales cargos docentes que prevé para el investigador.

${ }^{15}$ Asimismo, en la última convocatoria han sobresalido dos unidades ejecutoras o institutos CONICET en Bariloche: en primer lugar, el INIBIOMA (Universidad Nacional del Comahue y CONICET) con 12 ingresantes en 2020 -siendo el instituto CONICET con más ingresos a nivel nacional-; y por otro lado, el Nodo Bariloche del Instituto de Nanociencia y Nanotecnología (CNEA y CONICET) con 7 ingresantes a la CIC. 
Federalización e ingresos de investigadores a CONICET en 2019 y 2020... Págs. 233-269

${ }^{16}$ Lo que ocurre en estas universidades es que una de las grandes áreas del conocimiento no presenta el (alto) grado de desarrollo de las restantes.

${ }^{17}$ Según datos recopilados por el MINCYT (2018), si miramos en el agregado del periodo 2008-2017, el 65\% de los investigadores de la CIC que se encontraban en su primer año en el Consejo habían tenido previamente su beca doctoral y su beca postdoctoral en la misma institución en la que ingresaron como investigadores. Esa proporción muestra un mínimo de 47\% en el año 2016 y un máximo de $85 \%$ en 2012. 


\section{Referencias bibliográficas}

Aguilar Villanueva, L. (ed.) (1992) La hechura de las políticas. México DF: Miguel Angel Porrua.

Albornoz, M., R. Barrere, P. Sánchez Macchioli, L. Osorio y M. Turkenich (2015) Políticas CTI en países emergentes. Análisis comparado de experiencias heterogéneas y su aplicabilidad en Argentina. Informe de Proyecto de Investigación CIECTI-MINCYT.

Albornoz, M. y A. Gordon (2011) "La política de ciencia y tecnología en Argentina desde la recuperación de la democracia (1983-2009)". En M. Albornoz y J. Sebastián (eds.) Trayectorias de las políticas científicas y universitarias de Argentina y España. Madrid: CSIC: 67-22.

Aliaga,J. (2019) Algunas propuestas para el sistema de ciencia y técnica argentino. Documento disponible en: http://www.jorgealiaga.com.ar.

Aristimuño, F. y D. Aguiar (2015) “Construcción de las políticas de ciencia y tecnología en Argentina desde 1989 a 1999”. REDES 21(40):41-80.

Arleo, A., L. Sacco y R. Vidosa (2011) Relación entre capacitación y mercado de trabajo en ciencia y tecnología. La trayectoria del CONICET en el período 2004-2010. Ponencia presentada en el IV Encuentro regional de estudios del trabajo, Tandil, 16-17 de Junio.

Barceló, M., I. Abel, E. Fuster, N. Marrugat, A. Ordenes, N. Hernández, E. Santarremigia, A. Pujolar y D. Sánhez (2015) EU-Latin America cooperation on regional innovation strategies in the framework of regional policy. EU-LAC Foundation, European Union.

Barrera, M. (2019) Evolución de los salarios de los trabajadores del CONICET y de la Administración Pública Nacional durante la gestión de Cambiemos. Buenos Aires: Centro de Investigación y Formación de la República Argentina (CIFRA).

Beigel, F., O. Gallardo y F. Bekerman, F. (2018) "Institutional expansion and scientific development in the periphery: The structural heterogeneity of Argentina's academic field". Minerva 56(3):305-331.

Bekerman, F. (2016) "El desarrollo de la investigación científica en Argentina desde 1950: entre las universidades nacionales y el Consejo Nacional de Investigaciones Científicas y Técnicas”. Revista Iberoamericana de Educación Superior 7(18):3-23.

Botto, M. y L. Bentancor (2018) “Luces y sombras de la política de innovación científica y tecnología durante las gestiones kirchneristas (2003-2015)”. Revista Estado y Políticas Públicas 10:149-168. 
Britto, F.A., M. Pereira y G. Baruj (2014) Evaluación de programas públicos: Principales metodologías y experiencias de evaluación de programas de apoyo a la CTI en América Latina. Buenos Aires: Centro Interdisciplinario de Estudios en Ciencia,Tecnología e Innovación (CIECTI).

Caldelari, M., M. Casalet, E. Fernández y E. Oteiza (1992) "Instituciones de promoción y gobierno de las actividades de investigación”. En E. Oteiza (ed.) La política de investigación científica y tecnológica argentina. Buenos Aires: Centro Editor de América Latina:168-193.

Cao, H. y J. Vaca (2006) "Desarrollo regional en la Argentina: la centenaria vigencia de un patrón de asimetría territorial”. Revista EURE 32(95):95-111.

Casas, R., J.M. Corona y R. Rivera (2013) Políticas de ciencia, tecnología e innovación en América Latina: entre la competitividad y la inclusión social. Ponencia presentada en la Conferencia Internacional LALICS 2013, Río de Janeiro.

Casas, R. y J. Dettmer (2007) "Construyendo un paradigma de política científico tecnológica para México”. En J.L. Calva (ed.) Educación, ciencia, tecnología y competitividad. México DF: Miguel Angel Porrúa:137-154.

CEPAL (2017) Instituciones, políticas e instrumentos para impulsar la ciencia, tecnología e innovación en la Argentina: Reflexiones a partir de la experiencia brasileña. Santiago de Chile: CEPAL.

Chávez, M. (2008) La federalización del fomento a la ciencia, la tecnología y la innovación. Presentación en Seminario Fondos Mixtos: Evaluación y Prospectiva, Tepic, México.

CONICET (2006) Consejo Nacional de Investigaciones Científicas y Técnicas - 50 años. Buenos Aires: Edición Nacional Editora \& Impresora.

CONICET (2018) Informe de Gestión al 31-12-2018. Buenos Aires: CONICET.

Cruz Castro, L., P. Kreimer y L. Sanz Menéndez (2016) "Los cambios en los sistemas públicos de investigación de España y Argentina: el papel del CSIC y del CONICET en perspectiva comparada". En R. Casas y A. Mercado (eds.) Mirada iberoamericana a las políticas de ciencia, tecnología e innovación. Buenos Aires: CLACSO:73-104

Cruz Castro, L. y L. Sanz Menéndez (2018) “Autonomy and authority in public research organisations: Structure and funding factors”. Minerva 56:135-160.

Del Bello, J.C. (2007) “Contrarreforma (1990/96) y cambios en el CONICET a partir de 1996". En Ruptura y reconstrucción de la ciencia argentina. Buenos Aires: MINCYT-UNESCO:79-82. 
Del Bello, J.C. (2014) “Argentina: experiencias de transformación de la institucionalidad pública de apoyo a la innovación y al desarrollo tecnológico”. En G. Rivas y S. Rovira (eds.) Nuevas instituciones para la innovación: Prácticas y experiencias en América Latina. Santiago de Chile: CEPAL:35-78.

Del Bono, T. y M. Laborde (2020) Reflexiones sobre la Convocatoria 2020. Disponible en el Anexo I del Acta de Directorio del CONICET No. 519.

dos Santos, U.P. (2017) "Distribución espacial de los entes del sistema nacional de innovación brasileño: análisis de la década de 2000". Revista de la CEPAL 122:235-253.

Dye, T.R. (2017) Understanding public policy (15a. edición). Londres: Pearson.

Emiliozzi, S. y M. Unzué (2015) "La política de formación de recursos humanos altamente calificados en la Argentina reciente”. En S. Lago Martínez y N.H. Correa (eds.) Desafíos y dilemas de la universidad y la ciencia en América Latina y el Caribe en el siglo XXI. Buenos Aires: Editorial Teseo:293-303.

GACTEC (1999) Plan Nacional Plurianual de Ciencia y Tecnología 2000-2002. Buenos Aires: GACTEC.

Gallardo, O. (2015) “Trayectorias de formación de investigadores del CONICET". Revista Sociedad 34:121-139.

González, G. (2017) "Federalización de la ciencia y la tecnología en Argentina. Una revisión de iniciativas de territorialización y planificación regional (1996-2007)". Revista de Estudios Regionales 108:193-225.

González, G. y J. A. Claverie (2017) "Planeamiento de la Educación Superior en Argentina: Entre las Políticas de Regionalización y los Procesos de Innovación Universitaria (1995-2015)". Education Policy Analysis Archives 25(70):1-33.

González-López, M., B. Asheim y M.C. Sánchez-Carreira (2019) "New insights on regional innovation policies". Innovation: The European Journal of Social Science 32(1):1-7.

Gutti, P., Y. Kababe y F.Pizzarulli (2019) "La infraestructura científica y tecnológica en el sistema nacional de innovación”. En P. Gutti y C. Fernández Bugna (eds.) En busca del desarrollo: Planificación, financiamiento e infraestructuras en la Argentina. Bernal: Universidad Nacional de Quilmes:72-93. 
Houssay, B. (1960) "Importancia del adelanto científico para el desarrollo y la prosperidad de las Américas”. Ciencia Interamericana 1(1):11.

Hurtado, D. (2010) La ciencia argentina: Un proyecto inconcluso: 1930-2000. Buenos Aires: Edhasa.

Hurtado, D. (2016) “Ciencia para qué”. Revista Anfibia. Disponible en: http://revistaanfibia.com/ensayo/ciencia-para-que/.

Isaksen, A., R. Martin y M. Trippl, M. (2018) "New avenues for regional innovation systems and policy”. En A. Isaksen, R. Martin y M. Trippl (eds.) New avenues for regional innovation systems - Theoretical advances, empirical cases and policy lessons. Nueva York: Springer:1-19.

Jeppesen, C., M. Goldberg, A. Szpeiner y M.C. Rodríguez Gauna (2015) "Estrategias, instrumentos y resultados de la política pública de RRHH en los últimos diez años”. Revista Sociedad 34:68-84.

Jeppesen, C., M. Goldberg, A. Szpeiner, M.C. Rodríguez Gauna, R. Misiac y J. Silvani, (2016) "La formación de doctores en Argentina: Avances y desafíos desde la perspectiva CONICET”. Revista Argentina de Educación Superior 8(12):149-173

Laborde, M. y T. Del Bono (2019) Propuestas para comenzar a solucionar algunos de los principales problemas que afectan al CONICET. Buenos Aires: CONICET.

Lahera, P.E. (2006) "Encuentros y desencuentros entre políticas y políticas públicas". En R. Franco y J. Lanzaro (eds.) Política y políticas públicas en los procesos de reforma de America Latina. Buenos Aires: Miño y Dávila:75-99.

Lastra, K.F. (2017) "Investigación educativa en Argentina: impacto de las políticas de ciencia y tecnología en dos agencias del Estado, ANPCYT y CONICET”. Revista Iberoamericana de Educación Superior 8(21):94-108.

Llisterri, J.J. y C. Pietrobelli, C. (2011) "Los sistemas regionales de innovación: conclusiones y recomendaciones”. En J.J. Llisterri, y C. Pietrobelli (eds.) Los sistemas regionales de innovación en América Latina. Washington DC: Banco Interamericano de Desarrollo:104-122.

Losada Trabada, A. (2003) “Entre la ciencia política básica y la ciencia política aplicada: de la política a las políticas, del análisis a la gestión”. Revista de Investigaciones Políticas 2(1-2):63-81. 
Lugones, G., D. Hurtado, P. Gutti, E. Mallo, H. Bázque y M. Alonso (2010) El rol de las universidades en el desarrollo científico y tecnológico en la década 1998-2007: Informe Nacional Argentina. Santiago de Chile: Centro Interuniversitario de Desarrollo (CINDA) - Universia.

Marquina, M. y A. Chiroleu, A. (2015) “¿Hacia un nuevo mapa universitario? La ampliación de la oferta y la inclusión como temas de agenda de gobierno en Argentina". Propuesta Educativa 43:7-16.

Matera, R. (1992) Desafío aceptado: Pensamiento sobre la ciencia y tecnología contemporáneas. Buenos Aires: SECYT.

MINCYT (2011) Hacia una Argentina Innovadora: Plan Nacional de Ciencia, Tecnología e Innovación. Lineamientos 2012-2015. Buenos Aires: MINCYT.

MINCYT (2013) Argentina Innovadora 2020: Plan Nacional de Ciencia, Tecnología e Innovación. Lineamientos Estratégicos 2012-2015. Buenos Aires: MINCYT.

MINCYT (2018) Plan Argentina Innovadora 2030: Mesa de Recursos Humanos. Apéndice documental. Buenos Aires: MINCYT.

MINCYT (2020) Informe sobre infraestructuras de investigación en Argentina. Año 2, No. 2 Julio 2020. Buenos Aires: MINCYT.

Niembro, A. (2015) "Las brechas territoriales del desarrollo argentino: Un balance (crítico) de los años 2000”. Desarrollo Económico 55(215):21-47.

Niembro, A. (2020a) “¿Federalización de la ciencia y tecnología en Argentina? La carrera del investigador de CONICET (2010-2019)”. Ciencia, Docencia y Tecnología 31(60):1-33.

Niembro, A. (2020b) “¿Qué significa la federalización de la ciencia y tecnología en Argentina?”. Ciencia, Tecnología y Política 3(4):1-11.

Oszlak, O. y G. O’Donnell (1995) “Estado y políticas estatales en América Latina: hacia una estrategia de investigación”. REDES 2(4):98-128.

Peirano, F. (2011) “El FONTAR y la promoción de la innovación en empresas entre 2006 y 2010”. En F. Porta y G. Lugones (eds.) Investigación cientifica e innovación tecnológica en la Argentina: impacto de los fondos de la Agencia Nacional de Promoción Científica y Tecnológica. Bernal: Editorial Universidad Nacional de Quilmes:81-131. 
Rivas, G., S. Rovira y S. Scotto (2014) "Reformas a la institucionalidad de apoyo a la innovación en América Latina: antecedentes y lecciones de estudios de caso". En G. Rivas y S. Rovira (eds.) Nuevas instituciones para la innovación: Prácticas y experiencias en América Latina. Santiago de Chile: CEPAL:11-33.

Sadosky, M. (1984) Lineamientos de política científica y tecnológica. Buenos Aires: SECYT.

Sanz Menéndez, L., L. Cruz Castro, K.Jonkers, G. Derrick, M. Bleda y C. Martínez (2011) Public research organisations. Policy Brief, The Innovation Policy Platform, OECD.

Sarthou, N. (2019) “Tendencias en la evaluación de la ciencia en Argentina: género, federalización y temas estratégicos”. Ciencia, Docencia y Tecnología 30(59):37-73.

SECYT (2006) Plan Estratégico de Ciencia, Tecnología e Innovación Bicentenario (20062010). Buenos Aires: SECYT.

Stefani, F. (2018) Rol actual y futuro de la ciencia en la innovación industrial y el crecimiento económico en Argentina. Buenos Aires: Centro de Investigaciones en Bionanociencias (CIBION).

Suárez, D. y F. Fiorentin (2018) Federalización y efecto Mateo en la política científica: El caso del PICT en la Argentina (2012-2015). Buenos Aires: Centro Interdisciplinario de Estudios en Ciencia, Tecnología e Innovación (CIECTI).

Szpeiner, A. y C. Jeppesen (2013) Políticas de Federalización en Ciencia y Tecnología: Avances en el CONICET. Ponencia presentada en el VII Congreso Argentino de la Administración Pública, Mendoza, 18-20 de Septiembre.

Unzué, M. (2015) "Nuevas políticas públicas de formación de doctores en Argentina". Revista Sociedad 34:12-34.

Unzué, M. y S. Emiliozzi (2017) “Las políticas públicas de Ciencia y Tecnología en Argentina: un balance del período 2003-2015”. Temas y Debates 21(33):13-33.

Vargas Velásquez, A. (1999) Notas sobre el Estado y las políticas públicas. Bogotá: Almudena Editores. 


\section{Notas en periódicos digitales:}

Agencia Télam (03-08-2013). "El Conicet lanza incentivos para federalizar la investigación”. Disponible en: https://www.telam.com.ar/notas/201308/27459-el-conicetlanza-incentivos-para-federalizar-la-investigacion.html

Agencia Télam (18-01-2020). "Ana María Franchi: Queremos federalizar el Conicet y bajar la ciencia al territorio”. Disponible en: https://www.telam.com.ar/ notas/202001/425037-ana-maria-franchi-subrayo-que-hay-que-volver-a-poner-envalor-a-las-ciencias-sociales.html

Agencia TSS (19-09-2019). “El destino de la ciencia argentina”. Disponible en: http:// www.unsam.edu.ar/tss/el-destino-de-la-ciencia-argentina/

Agencia TSS (24-01-2020). "Diego Hurtado: En la Argentina la ciencia es potente pero la tecnología es débil". Disponible en: http://www.unsam.edu.ar/tss/ diego-hurtado-en-la-argentina-la-ciencia-es-potente-pero-la-tecnologia-es-debil/

Diario Contexto(03-07-2017)."ElcandidatocientíficoqueUnidadCiudadana eligiópara llevar al Congreso". Disponible en: https://www.diariocontexto.com.ar/2017/07/03/ el-candidato-cientifico-que-unidad-ciudadana-eligio-para-llevar-al-congreso/

Diario La Gaceta (09-07-2017). "Federalizar el Conicet es una necesidad, por una cuestión de equidad”. Disponible en: https://www.lagaceta.com.ar/nota/736610/actualidad/federalizar-conicet-necesidad-cuestion-equidad.html

Diario Página12 (12-05-2019).“Conmociona advertir hasta dónde llegamos”.Disponible en: https://www.pagina12.com.ar/193210-conmociona-advertir-hasta-donde-llegamos

Diario Página12 (15-03-2017). "El Conicet en debate”. Disponible en: https://www. pagina12.com.ar/25740-el-conicet-en-debate

Portal AgendAR (10-10-2019). "Roberto Salvarezza propone "Argentina, sociedad del conocimiento»". Disponible en: https://agendarweb.com.ar/2019/10/10/ argentina-sociedad-del-conocimiento/

Portal Política Argentina (02-10-2019). "El gobierno de Macri puso en jaque a la ciencia: el diagnóstico del referente de Alberto en CyT en Diputados". Disponible en: https://www.politicargentina.com/notas/201910/30714-el-gobierno-de-macri-pusoen-jaque-a-la-ciencia-el-diagnostico-del-referente-de-alberto-en-cyt-en-diputados.html 
Portal UNERnoticias (07-05-2019). “Apostar a un CONICET que lidere, estimule y articule la investigación y la ciencia en Argentina”. Disponible en: https://noticias. uner.edu.ar/entrevistas/7173/apostar-a-un-conicet-que-lidere-estimule-y-articule-lainvestigacion-y-la-ciencia-en-argentina

\section{Cómo citar este artículo}

Niembro, Andrés, Francisco Aristimuño y Juan Carlos Del Bello (2021) "Federalización e ingresos de investigadores a CONICET en 2019 y 2020: ¿Del dicho al hecho hay mucho trecho?”. Revista Perspectivas de Políticas Públicas vol. 10 No 20:233-269 